\title{
A Retina-Based Perceptually Lossless Limit and a Gaussian Foveation Scheme With Loss Control
}

\author{
André Luiz N. Targino da Costa, Member, IEEE, and Minh N. Do, Fellow, IEEE
}

\begin{abstract}
In this paper, we propose a new limit that promises theoretically achievable data reduction ratios up to approximately 9:1 with no perceptual loss in typical scenarios. Also, we introduce a novel Gaussian foveation scheme that provides experimentally achievable gains up to approximately 2 times the compression ratio of typical compression schemes with less perceptual loss than in typical transmissions. Both the proposed limit and foveation scheme shares the same background material: a model of image projection onto the retina; a model of cones distribution; and, subsequently, a proposed pointwise retina-based constraint called pixel efficiency. Quantitatively, the lattermost is globally processed to reveal the perceptual efficiency of a display. Analytical results indicate that in general the perceptual efficiency of displays are low for typical image sizes and viewing distances. Qualitatively, the pixel efficiency is used together with a lossy parameter to locally control the spatial resolution of a foveated image. Practical results show that proper use of the lossy parameter in the foveation filtering can increase the subjective quality of images.
\end{abstract}

Index Terms-Compression, foveation, image processing, perception.

\section{INTRODUCTION}

$\mathbf{K}$ NOWLEDGE of human perception has always been an object of desire. Through the ages, humankind has sought for answers about how we perceive the world. Along with the evolution of science, a substantial number of theories arose trying to explain how our different modalities of perception work. However, due to lack of supporting evidence, many theories were considered erroneous. One good example is Hering's Color Opponency theory [1]. After the successful outcome of Young's Trichromatic theory [2], Hering's theory fell in disbelief ${ }^{1}$.

In order to give grounds for a perception related theory and avoid discredit, three complementary approaches can be taken [3]: psychological; biological; or theoretical. Typically, engineering studies use mostly the third method to justify their works, and, whenever it is necessary, utilize results provided by the other two to make a point satisfactorily clear.

Manuscript received August 30, 2013; revised February 01, 2014; accepted March 07, 2014. Date of publication April 04, 2014; date of current version May 13,2014. The guest editor coordinating the review of this manuscript and approving it for publication was Prof. Homer Chen.

The authors are with the Department of Electrical and Computer Engineering, University of Illinois at Urbana-Champaign, Urbana, IL 61801 USA (e-mail: targino1@illinois.edu; minhdo@illinois.edu).

Color versions of one or more of the figures in this paper are available online at http://ieeexplore.ieee.org.

Digital Object Identifier 10.1109/JSTSP.2014.2315716

${ }^{1}$ Relatively recent studies consider that both theories are actually correct, they just take place in different stages of the human visual processing.
Our main objective in this paper is to put some of those theories together and, therefore, present and develop new models, limits, and filters, regarding the Human Visual System (HVS). More specifically, we want to find a perceptually efficient way of representing images and determine its theoretical limitations. In order to achieve such objective, we need to have a good understanding of the human visual perception [3], [4].

The main motivation behind such objective is unfolded when we recognize the substantial contributions it can give to many different applications. Some notorious examples of such point of view are: the eyeglasses [5], [6] (which exploits a good understanding and modeling of the eye optics); the halftone printing [7], [8] (which exploits a good understanding and modeling of the eye resolution); and the television [9], [10] (which also exploits a good understanding and modeling of the eye resolution in addition to a good understanding of the trichromatic theory). The usefulness of those inventions reveals the relevance of our research.

In terms of practical applications, our findings could be used for many different purposes, including: enhancing the quality and efficiency of videoconferencing; improving human-like visual tasks performed by computer vision algorithms; and, as we will show later, boosting the performance of video compression schemes.

In order to find a perceptually efficient way of representing images, this paper make use of a technique known as foveation [11]. Such technique exploits the fact that, in human color vision, the visual resolution decays spatially from a spot in the human retina called fovea. This principle can promote the reduction of image resolution through the use of a shift-varying low-pass filter in a process called foveation filtering. Typically, foveation is used to improve video quality in low bit rate transmissions [12]-[15]. However, it is unclear whether or not there is a way to quantify how much one image can be compressed with no perceptual loss.

Our main contribution in this paper is as follows. A new limit (perceptual efficiency) to precisely quantify the maximum sampling rate reduction based on foveation with no perceptual loss (we also compute this perceptual efficiency for a number of recent displays to show the potential gain by such resampling). A new foveation scheme with adjustable parameter to control the amount of foveation-based information reduction in lossy compression, depending on the target amount of bits. Extensive subjective testing with 1080p frames to verify the perceptual lossless property and gain in lossy coding of our proposed methods in comparison with standard coding and other foveation coding schemes.

Details about the derivation of the limit and of the foveation scheme, together with some additional contributions, are provided throughout the text. We believe the present study is very 


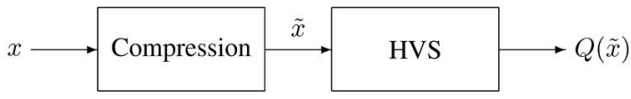

(a) Typical scenario.

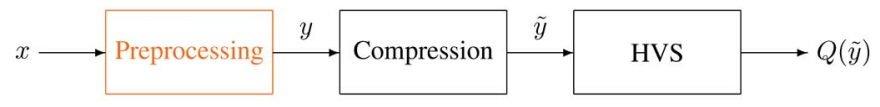

(b) Proposed solution.

Fig. 1. Perceptual quality of compressed images. (a) Typical scenario. (b) Proposed solution.

timely since display resolutions increase in a fast pace [16], [17] and, as we will show later, the higher the resolution, the more valuable the presented work.

The outline of this paper is as follows. Section II provides the problem statement. Section III makes a review of related work. Section IV shows the necessary background to understand our work. Section V describes the proposed material which includes: a model of image projection onto the retina; a model of cones distribution; perceptual limits; and foveation filters. Section VI presents a comparison between foveation filtering methods. Section VII discusses important points of our results. Section VIII summarizes the conclusion of the paper. Finally, Section IX exposes our plans for future work.

\section{Problem Statement}

Let $Q(x)$ denote the perceptual quality of an image (or frame) $x$, and $\tilde{x}$ denote an approximation of $x$. In Fig. 1(a), $x$ is encoded with an encoding bit rate and, due to lossy compression, $\tilde{x}$ is decoded. Then, the decoded approximation is processed by the HVS and its perceptual quality is assessed. The problem is that, in practice, limits on encoding bit rates significantly affect the quality of $\tilde{x}$. As a result, $Q(\tilde{x})<Q(x)$, and often $Q(\tilde{x}) \ll Q(x)$.

In order to solve this problem, we propose a solution shown in Fig. 1(b). Our objective is to preprocess $x$ by reducing its amount of data as much as possible so that, with no increase in the bit rate, $Q(\tilde{y})>Q(\tilde{x})$ and (ideally) $Q(y)=Q(x)$.

The question now is whether such solution is possible. In order to answer that question, we can split it into three parts:

1) Is it possible to preprocess an image $x$ yielding an image $y$ so that $Q(y)=Q(x)$ ?

2) If so, how much data in the image $x$ can be reduced this way?

3) Finally, can such preprocessing make $Q(\tilde{y})>Q(\tilde{x})$ without increasing the bit rate?

This paper is devoted to address these three questions and to show through theoretical and practical results that such achievements are possible.

\section{RELATED WORK}

Arguably the first work on foveation was done in [18], where an image is first decomposed into a multiresolution image pyramid and then reconstructed with different resolutions for different parts of the image. The idea of locally varying the spatial resolution of an image was employed shortly after in a few applications, including: sensing [19], [20], where some space-variant visual sensor schemes based on foveation are proposed; displaying [21], by means of a description of how to acquire and display foveated images with shift-varying sampling densities; visual recognition [22], through the use of a framework with only two levels of details; rendering [23], performed with only three different resolutions; and image compression [24], [25], where the former uses a log polar mapping which resamples imagery at peripherally decreasing resolution, and the latter utilizes a foveation criterion to locally select spatial resolution for image coding.

In terms of applications, video compression is the one that has received the most attention in the literature over the years. Basu et al. [26] were among the first to propose using spatially varying sampling due to foveation to improve image and video compression. Another contribution to the field can be found in [12], where a neurobiological model of visual attention is applied to automatically select regions of high saliency in videos. Such technique can be combined with virtually all foveation methods to promote automatic prediction of gaze, including ours. Another substantial contribution was given in [13], where a wavelet-based method is employed to perform scalable video coding. Such method was even further extended in [14], when it was combined with an automated foveation point selection scheme and an adaptive frame prediction algorithm.

The previous method, however, is often referred as a multiresolution method, whereas ours is based on foveation filtering. One of the major contributions given by a filtering-based method is found in [15], where foveation is exploited as a tool for exploring methods for optimal rate control of foveated video. Such work is based on a foveation scheme previously described in [27], which is further used by Lee et al. as an inspiration to achieve several different goals, including the development of: methods for foveated image and video quality assessment [28]; efficient algorithms for foveated image and video compression [29]; foveation-based error resilience and unequal error protection schemes [30]; and an end-to-end foveated visual communication system [31]. Ho et al. [32] propose to use a foveation model to reduce rate of a compressed video.

Recently, foveation principles have been used to achieve some fairly distinctive goals. In [33], the falloff of acuity in the visual periphery is exploited to accelerate graphics computation. In [34], a novel algorithm attention emphasized bit arrangement approach for human vision on wavelet-based video coding is proposed. A more extensive exploitation of perceptual features is observed in [35], where a human perceptual visual quality-oriented saliency is proposed. Such saliency takes into account the influences of the visual stimuli, the limited human capacity of spatial-temporal resolution, and the visual sensory memory collectively.

Particularly, we believe better results can be achieved when a more complete and accurate information regarding the HVS is taken into consideration. One good example of such thinking is found in [36], where a Gaussian foveation method, based on the receptive fields of ganglion cells, improves upon a graph-based method [37] in terms of the quality of image reconstruction. Examples like this show that not only knowledge regarding photoreceptors distribution [38] should be used, but also information about subsequent layers of the HVS, such as retinal ganglion cells distribution [39], [40] and receptive fields sizes [40], should be considered. 


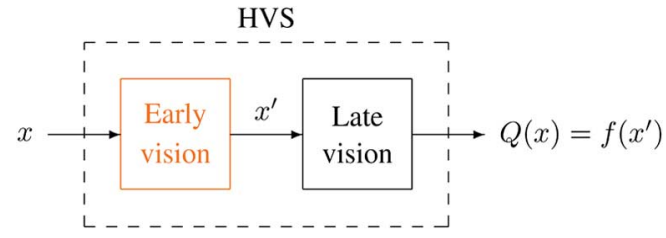

Fig. 2. Subdivision of the HVS into early vision and late vision.

Finally, we are not aware of any studies which report whether or not there is a way to quantify how much one image can be compressed with no perceptual loss.

\section{BACKGROUND}

The HVS is known to be one of the most complex structures in the human brain. Among all of the five senses, sight is probably the most complex one. Consequently, many different subdivisions can be found in the literature regarding the human visual processing. Here, we consider the subdivision between early vision and late vision. Early vision can be defined as all the stages of visual processing up to and including the primary visual cortex [41], whereas late vision as all the stages of visual processing after the primary visual cortex. A block diagram that expresses this idea can be seen in Fig. 2.

Such block diagram gives us an important clue on how to answer the first question on our problem statement. Although the HVS assesses the perceptual quality of $x$, such perceptual quality is actually a function of $x^{\prime}$ (considering no feedback in the model).

It is clear that a better understanding of how early vision works is desired to address the questions we are concerned about. It is known [3] that the first stage of the human visual processing occurs on the photoreceptor cells at the back of the human retina and a later stage of the early vision occurs on the retinal ganglion cells.

Regarding the first stage, one can basically separate the photoreceptors into two groups: cones and rods. Rods are very sensitive to light, so they cease responding if light intensity reaching them is too high [3]. In particular, we will only deal with cones, since the applications we will make use of provide light intensities higher than what is necessary to cease the rods response.

Regarding the later stage, each retinal ganglion cell gathers the signals transmitted by the cones located in its receptive field [3]. It is important to know that the number of cones in each receptive field is not the same for all retinal ganglion cells. Also, both the distribution of cones and the distribution of retinal ganglion cells are not uniform across the human retina.

Cones are mostly concentrated in the fovea. When staring at a point, the projection onto the retina of such point lies in the fovea. Also, the higher the distance from the fovea, the less the number of cones (for the most part of it). Such distance is commonly referred in the literature as eccentricity, and it is usually measured in terms of degrees or millimeters. In particular, we will always measure the eccentricity in terms of millimeters.

\section{Proposed Material}

We start the proposed material addressing the first question of our problem statement. Specifically, if an image $x$ is preprocessed yielding an image $y$ such that $x^{\prime}=y^{\prime}$, i.e., the early vision output of the former equals the early vision output of the latter, then $Q(x)=Q(y)$. This can be verified by inspection of Fig. 2 .

Now, in order to understand how can we preprocess an image in such a way, we need to understand first how an image is projected onto the retina.

\section{A. Model of Image Projection Onto the Retina}

We want to map every point in the viewing scene to a point on the retina. After making the map, we need to perform a nonuniform sampling of the image projected onto the retina. So, we have a problem of non-uniform sampling of a 2-D continuous signal.

In order to transform this problem into an easier problem, we can exploit the fact that a uniform sampled version of a 2-D continuous signal completely describes such signal (once the Nyquist rate is respected when performing the sampling). Yet, we know that in practice the 2-D continuous signal is not bandlimited, nor is the sampling frequency above the Nyquist rate. However, for practical reasons, it is quite reasonable to assume that in general such sampling process does not yield significant enough aliasing (to be noticed by the HVS) in the spectrum of the discrete version of the 2-D continuous signal.

Now, we have a problem in which we are given a set $x$ of regular samples of a 2-D continuous signal from which we wish to obtain amplitudes for a set $x^{\prime}$ of irregularly spaced samples, i.e., we have a resampling problem from a regular grid to an irregular grid. Zeevi and Shlomot [42] show that this problem can be solved by using a projection filter $f_{R}$ (that means $\left.f_{R}(x)=f_{R}\left(f_{R}(x)\right)\right)$ to the space of locally bandlimited signals corresponding to the irregular sampling grid to obtain $x^{\prime}=R\left\{f_{R}(x)\right\}$, where $R\{\cdot\}$ denotes a resampling operator. Consequently, assuming that the HVS performs such projection filtering in the first stage of the human visual processing (i.e, $\left.f_{H V S}=f_{R}\right)$, then from Fig. 2 we have $Q(x)=Q\left(f_{R}(x)\right)$ since $f_{H V S}(x)=f_{H V S}\left(f_{R}(x)\right)$. Thus, we address the first question of our problem statement, i.e. we can preprocess an image $x$ yielding an image $y$ so that $Q(y)=Q(x)$.

So, before proceeding to the design of the desired filter, we need to understand how the samples of the 2-D continuous signal are projected onto the retina. Here, we consider that the human eye is shaped like a perfect sphere with retinal radius of $11.06 \mathrm{~mm}$, as in [38]. Also, we assume that the image plane is perpendicular to the line that passes through the fovea and the point we are staring at, as illustrated in Fig. 3. Consequently, the distance $d_{D}\left(l_{1}, l_{2}\right)$ between $P^{\prime}$ (the projection onto the retina of point $P$ ) and the fovea (the projection onto the retina of point $S$ ) can be given by:

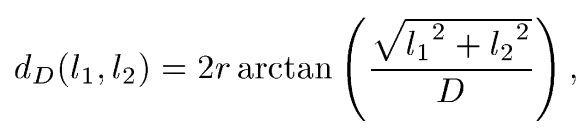

where $r=11.06 \mathrm{~mm}, \arctan (\cdot)$ is in radians, $l_{1}$ and $l_{2}$ are the distances between $P$ and $S$ projected onto the horizontal and vertical axis, respectively, and $D$ is the viewing distance.

Equivalently, $d_{D}\left[p_{1}, p_{2}\right]$ can be given by:

$$
d_{D}\left[p_{1}, p_{2}\right]=2 r \arctan \left[\frac{1}{D} \sqrt{\left(\frac{p_{1}}{\Gamma_{1}}\right)^{2}+\left(\frac{p_{2}}{\Gamma_{2}}\right)^{2}}\right],
$$




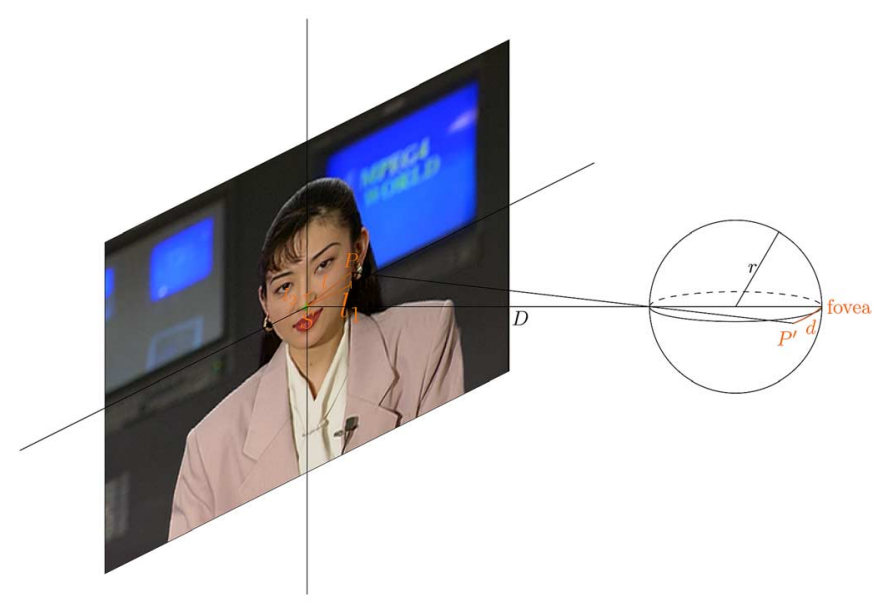

Fig. 3. Model of image projection onto the retina.

where $p_{1}$ and $p_{2}$ are the distances (in pixels) between $P$ and $S$ projected onto the horizontal and vertical axis, respectively, $\Gamma_{1}$ and $\Gamma_{2}$ are the horizontal and the vertical pixel densities of the display (in pixels per unit of length of $D$ ), respectively, and everything else is defined as before.

The proposed model of image projection onto the retina considers that there is only one point where the light passes through the eye, and that such point, $P$, and $P^{\prime}$ are three collinear points. Such considerations, together with our previous ones, can be used to show that there is a bijection between the set of all the points in the image plane and the set of all the points in the retina. In order to understand the form of such bijection, we can use the following demonstration.

Let $l$ be the distance between $P$ and $S$, and let everything else be defined as before. Then the distance between $P^{\prime}$ and the fovea is given by $d_{D}(l)=2 r \arctan (l / D)$. Now, consider the set of points $P$ which are all the same distance $l$ from $S$, i.e., a circle of radius $l$ in the image plane. For every point of such set there is a point $P^{\prime}$ in the retina whose distance from the fovea is given by the same distance $d_{D}(l)$. Hence, the locus of all $P^{\prime}$ is also a circle and it lies on a plane parallel to the image plane. This means that there is a bijection between the circle in the image plane and the circle in the retina, since they can be seen as two sections of the same conic. Now, consider the set of all the possible circles in the image plane centered at $S$, which can be called concentric set. By the same reasoning, there exists a bijection between any circle of radius $l$, where $0 \leq l<\infty$, in the concentric set and a circle in the retina whose points are all the same distance $d_{D}(l)$ from the fovea. The set of all such circles in the retina can be called parallels of longitude, since they lie on planes parallel to the image plane. Now, since the function $\arctan (\cdot)$, with domain $[0, \infty)$ and codomain $[0,(\pi / 2))$, is a bijective function, then there is a bijection between the concentric set and the parallels of longitude. Also, since $0 \leq l<\infty$, then $0 \leq d_{D}(l)<\pi r$, which is exactly the set of all the possible distances between a point in the retina and the fovea (the point where $d_{D}(l)=\pi r$ is not in the retina since it represents the pupil,i.e., the point where the light passes through the eye). This means that there is a bijection between the set of all the points of all the circles in the concentric set and the set of all the points in the retina. Finally, since the set of all the points of all the circles in the concentric set is actually the set of all the points in the image plane, then there is a bijection between the set of all the points in the image plane and the set of all the points in the retina.

From the preceding demonstration we can see that a circle in the image plane centered at $S$ will be projected to a circle in the retina centered at the fovea. This means that the only spatial information from a pixel $P$ that is relevant for us is actually its distance from $S$. Also, such demonstration can be used to show how a non-linear mapping from the image plane to the retina behaves in terms of such distance.

In practice, however, a pixel is not an ideally impulsive source of light that is projected onto a point at the retina. Instead, it has a support region and it is projected onto an area at the retina containing a certain number of cones. Hence, considering a regular 2-D grid of rectangular pixels, the area $A_{D}\left[p_{1}, p_{2}\right]$ of the projection onto the retina of a pixel centered at $P$ can be approximated by:

$$
\begin{aligned}
A_{D}\left[p_{1}, p_{2}\right] & \approx 4 r^{2}\left[\arctan \left(\frac{p_{1}+0.5}{D \Gamma_{1}}\right)-\arctan \left(\frac{p_{1}-0.5}{D \Gamma_{1}}\right)\right] \\
\times & {\left[\arctan \left(\frac{p_{2}+0.5}{D \Gamma_{2}}\right)-\arctan \left(\frac{p_{2}-0.5}{D \Gamma_{2}}\right)\right], }
\end{aligned}
$$

where everything is defined as before.

Now, in order to find the number of cones in such area, we need to understand first how the cones are distributed over the retina.

\section{B. Model of Cones Distribution}

We want to find the areal number density $n_{c}$ of cones per square millimeter in every point on the retina. Since we consider that the human eye is shaped like a perfect sphere, we can make use of a spherical coordinate system to determine $n_{c}$. In this case, the coordinate surface is a sphere with radius $r$, and consequently $n_{c}$ is a function of only two variables.

It is possible to go even further and consider $n_{c}$ to be a function of the eccentricity only. Therefore, based on a study with a vast number of measurements performed on human subjects [38], we believe that, when drawn directly from such study, the areal number density $n_{c}(d)$ of cones per square millimeter can be approximated by:

$$
n_{c}(d) \approx k_{c_{1}} \lambda e^{-\lambda d}+k_{c_{2}}, \quad d \geq 0
$$

where $k_{c_{1}}=45000, k_{c_{2}}=5000, \lambda=0.5$, and $d$ is the eccentricity (in millimeters).

Fig. 4 shows the areal number density $n_{c}$ of cones per square millimeter plotted as a function of eccentricity.

It follows that the precise number of cones per pixel $q_{D}\left[p_{1}, p_{2}\right]$ of a pixel centered at $P$ can be given by:

$$
q_{D}\left[p_{1}, p_{2}\right]=\iint_{S_{P}} n_{c} \mathrm{~d} S,
$$

where $S_{P}$ is the surface defined by the projection onto the retina of a pixel centered at $P, \mathrm{~d} S$ is a surface element, and everything else is defined as before.

In order to make the task of calculating $q_{D}\left[p_{1}, p_{2}\right]$ easier, we can assume that over a small surface $S_{P}$ on the retina, $n_{c}$ vari- 


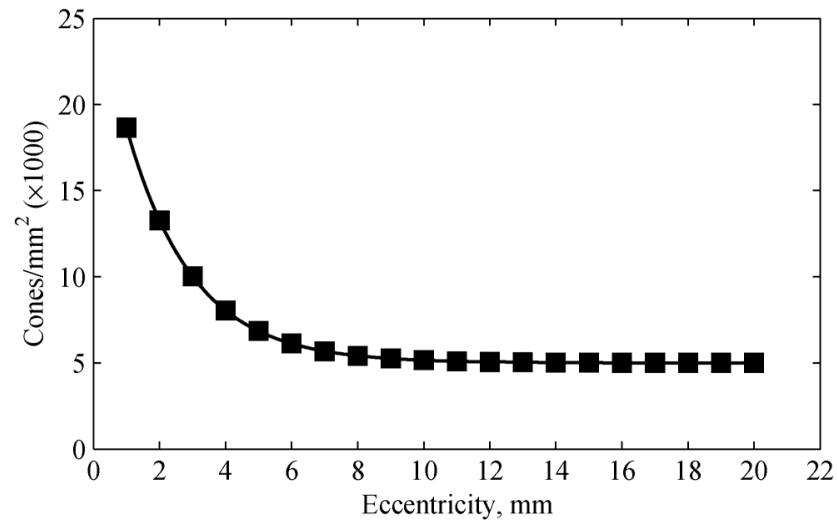

Fig. 4. Model of cones distribution.

ates little and can be approximated by $n_{c}\left(d_{D}\left[p_{1}, p_{2}\right]\right)$ at all the points of such surface. So:

$$
q_{D}\left[p_{1}, p_{2}\right] \approx n_{c}\left(d_{D}\left[p_{1}, p_{2}\right]\right) \iint_{S_{P}} \mathrm{~d} S
$$

Also, we can use (2) to approximate $A_{D}\left[p_{1}, p_{2}\right]=\iint_{S_{P}} \mathrm{~d} S$, i.e., the area of the projection onto the retina of a pixel centered at P. Hence, the number of cones per pixel $q_{D}\left[p_{1}, p_{2}\right]$ of a pixel centered at $P$, can be approximated by:

$$
q_{D}\left[p_{1}, p_{2}\right] \approx n_{c}\left(d_{D}\left[p_{1}, p_{2}\right]\right) A_{D}\left[p_{1}, p_{2}\right]
$$

where $d_{D}\left[p_{1}, p_{2}\right]$ is given by (1), $A_{D}\left[p_{1}, p_{2}\right]$ is given by (2), and $n_{c}(d)$ is given by (3).

\section{Perceptual Limits}

We want to give an answer to the second question of our problem statement. In order to do so, we could find a ratio between the total number of cones stimulated by an image and the total number of pixels in such image. This ratio would give us an idea of what fraction of the total number of pixels we would be able to fully process in our HVS.

In order to find such ratio, we could use the aforementioned bijection, between the set of all the points in the image plane and the set of all the points in the retina, to guarantee there would be neither overlaps nor gaps among the projections onto the retina of all pixels in an image. Then we could sum the number of cones per pixel $q_{D}$ over all pixels in that image to find the total number of cones stimulated by such image. Finally, we could divide such number by the total number of pixels in the image to find the desired ratio.

However, we believe this ratio would not be a good measurement of efficiency, since we can have images where such ratio is bigger than 1 while having many pixels not fully processed by the HVS, i.e., many pixels where $q_{D}<1$. In order to overcome this issue, we propose the following material.

As it can be seen from the proposed model of image projection onto the retina, in one given image there are possibly some pixels that contain more information than the HVS can process. This means that possibly such image representation is not $100 \%$ perceptually efficient. More specifically, if a pixel is projected onto an area at the retina with one or more cones, then that pixel is $100 \%$ perceptually efficient. On the other hand, if two or more pixels are projected onto an area at the retina with just one cone, then those pixels cannot be totally processed by the HVS.

Based on that, we can use the number of cones which a pixel is projected onto as a measure of the efficiency of such pixel. Hence, we can say that the perceptual efficiency of a pixel can be determined by $q_{D}\left[p_{1}, p_{2}\right]$. Formally, the pixel efficiency $e_{D}\left[p_{1}, p_{2}\right]$ of a pixel centered at $P$ can be defined as:

$$
e_{D}\left[p_{1}, p_{2}\right] \triangleq \begin{cases}1, & \text { if } q_{D}\left[p_{1}, p_{2}\right] \geq 1 \\ q_{D}\left[p_{1}, p_{2}\right], & \text { else, }\end{cases}
$$

where $q_{D}\left[p_{1}, p_{2}\right]$ is given by (4).

The pixels where $e_{D}=1$ are located around $S$, i.e., the point where we are staring at. We call the image formed only by such pixels as macular image, inspired by the fact that macula is the region where cone photoreceptors are concentrated [3]. A macular image represents a section of an image whereas an image in curvilinear coordinates [15] represents a whole image. There is no coordinate transformation in the process of generating a macular image. The main purpose of a macular image is to enable the visualization of which pixels in an image we are able to fully process in our HVS. The size of the macular image will basically depend on the viewing distance $D$ and on the horizontal and the vertical pixel densities $\Gamma_{1}$ and $\Gamma_{2}$, respectively, of the display.

Fig. 5 shows the macular images and the pixel efficiencies of every pixel, where the pixel efficiency of a pixel can vary from 0 (displayed as black) to 1 (displayed as white), of an image of resolution $352 \times 288$, for a display of pixel density $\Gamma_{1}=\Gamma_{2}=$ 200 pixels per inch (PPI), and $S$ at coordinates $(176,144)$, under four different viewing distances $D$ in inches.

Based on the work presented so far, we can now propose a limit that assesses the efficiency of a display under a perceptual point of view. This limit considers the best case scenario of such efficiency, i.e., $S$ is considered to be at the center of the image. Formally, the perceptual efficiency $E_{\Gamma_{1} \times \Gamma_{2}, N_{1} \times N_{2}}(D)$ of a display of pixel density $\Gamma_{1} \times \Gamma_{2}$ that can display an image of resolution up to $N_{1} \times N_{2}$, and it is viewed at a distance $D$ can be defined as:

$$
\begin{aligned}
& E_{\Gamma_{1} \times \Gamma_{2}, N_{1} \times N_{2}}(D) \\
& \triangleq \frac{1}{N_{1} N_{2}} \sum_{n_{1}=1}^{N_{1}} \sum_{n_{2}=1}^{N_{2}} e_{D}\left[\left|n_{1}-\left\lceil\frac{N_{1}}{2}\right\rceil\right|,\left|n_{2}-\left\lceil\frac{N_{2}}{2}\right\rceil\right|\right],
\end{aligned}
$$

where $e_{D}\left[p_{1}, p_{2}\right]$ is given by (5).

Inspection of (6) shows that the value range of the perceptual efficiency $E$ is given by $0<E \leq 1$. Also, such limit, together with our previous considerations, answers the second question of our problem statement. More precisely, the data in the image $x$ can be reduced to $E$ times its original amount with no visual perceptual loss. So, for example, an image of resolution $N_{1} \times$ $N_{2}$, i.e., an image containing $N_{1} N_{2}$ samples can be processed and reduced to $E N_{1} N_{2}$ samples with no perceptual loss. Finally, it is important to notice that such limit is actually an upper bound of the actual HVS limit, since later stages of the human visual 


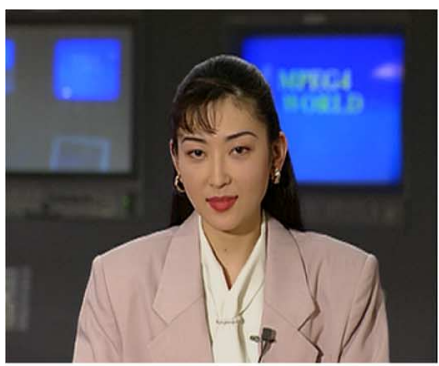

(a) Macular image for $D=10$.

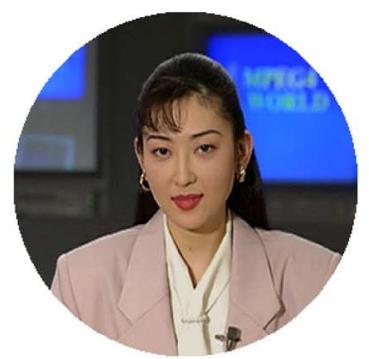

(b) Macular image for $D=15$. (c) Macular image for $D=17.5$ (d) Macular image for $D=20$.

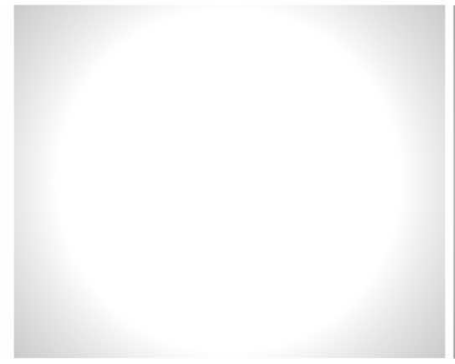

(f) Pixel efficiency for $D=15$.

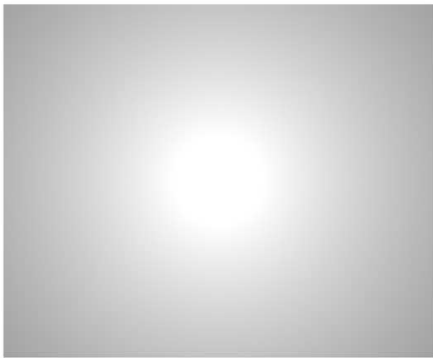

(g) Pixel efficiency for $D=17.5$.

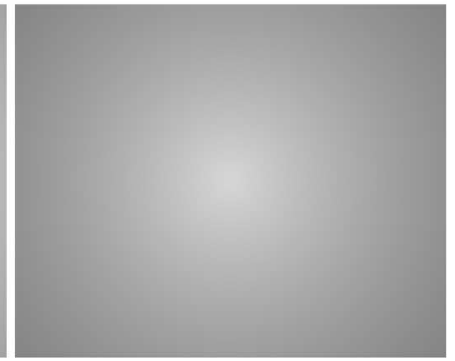

(h) Pixel efficiency for $D=20$.

Fig. 5. Macular images and the pixel efficiencies of every pixel of an image of resolution $352 \times 288$, for a display of pixel density $\Gamma_{1}=\Gamma_{2}=200$ PPI, and $S$ at coordinates $(176,144)$, under four different viewing distances $D$ in inches. (a) Macular image for $D=10$. (b) Macular image for $D=15$. (c) Macular image for $D=17.5$. (d) Macular image for $D=20$. (e) Pixel efficiency for $D=10$. (f) Pixel efficiency for $D=15$. (g) Pixel efficiency for $D=17.5$. (h) Pixel efficiency for $D=20$.

processing can reduce even more the amount of information we can process from an image.

Fig. 5 considers a display of density $\Gamma_{1}=\Gamma_{2}=200$ (PPI) presenting images of resolution $352 \times 288$. If we consider such image size as the maximum resolution of the display, then $N_{1}=$ 352 and $N_{2}=288$. In this case, the perceptual efficiency of the display at viewing distances $D=10 \mathrm{in}, D=15 \mathrm{in}, D=17.5$ in, and $D=20$ in would be approximately $100 \%, 97 \%, 81 \%$, and $65 \%$, respectively.

Finally, in a special case when $\Gamma_{1}=\Gamma_{2}=\Gamma$ and $N_{1}=$ $N_{2}=N$, the perceptual efficiency $E_{\Gamma_{1} \times \Gamma_{2}, N_{1} \times N_{2}}(D)$ given by (6) can be expressed simply as $E_{\Gamma, N}^{s}(D)$ and defined as:

$$
E_{\Gamma, N}^{s}(D) \triangleq E_{\Gamma \times \Gamma, N \times N}(D) \text {. }
$$

\section{Foveation Filters}

As mentioned before, the main motivation for the use of the proposed filters is that, according to [42], the problem of resample from a regular grid to a irregular grid can be solved by using a projection filter. Also, it is important to notice that the information about $D$ and $S$ should be available in order to properly design such a filter.

Specifically, the task of finding $D$ can be considered an easier assignment than determining $S$, and can be done through the use of depth cameras for example. The consideration of tracking for fixation point $S$ that may be a key factor of the proposed method deserves considerably more attention. In order to solve that issue we could, as mentioned before, promote automatic prediction of gaze by combining our method with a neurobiological model of visual attention that is applied to automatically select regions of high saliency in videos [12]. Alternatively, we could use eye tracking systems to determine $S$ since, as mentioned in [15], several real-time/nonreal-time visual communication systems associated with eye trackers have already been proposed and demonstrated in the field of visual communications as well as virtual reality. Finally, we could even provide such information about $D$ and $S$ by the manual use of a mouse.

In this paper however, it is assumed that the information about $D$ and $S$ is available. The decision of which filter is going to be used is what plays a major role on the overall purpose of this paper. With that in mind, a few reasons led us to choose a Gaussian filter.

First, striate cells behave much as would be predicted if the receptive fields approximated a two-dimensional Gabor function [43]. This indicates that a Gaussian filter is well-suited to filter the image in a way that the whole human visual processing can be preserved.

Second, Gaussians provide the largest amount of low-pass filtering for the smallest spatial support (modulated Gaussians have a minimum joint time-frequency localization), thus making Gaussians ideal in terms of minimizing computations for the same bandwidth [36].

Third, Gaussian filters can be both separable (which helps improving the implementation regarding the computational time) and isotropic (which according to [27] can provide more symmetric frequency response associated with the local bandwidth).

Regarding the design of the filter itself, we use a 2-D discrete shift-varying Gaussian filter with: zero mean; standard deviation $\sigma_{p_{1} p_{2}}=-0.38 \ln e_{D}\left[p_{1}, p_{2}\right]$ at $P$ (see Appendix A for details); and a squared support region centered at $P$ of size $K_{p_{1} p_{2}} \times K_{p_{1} p_{2}}$ varying from $-\left\lceil 3 \sigma_{p_{1} p_{2}}\right\rceil$ to $\left\lceil 3 \sigma_{p_{1} p_{2}}\right\rceil$ in both vertical and horizontal directions. Such filter is applied in each component of a color image.

We also consider a case where visual perceptual loss is allowed. In this case, a more complete version of the low-pass shift-varying filter includes a lossy parameter $\Delta$, such that $\Delta \geq$ 0 . The inclusion of such parameter is inspired on the fact that the HVS reduces the amount of visual information not only in the 


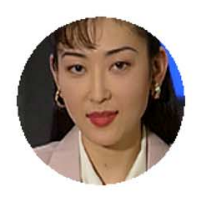

(a) Macular image for $\Delta=1$.

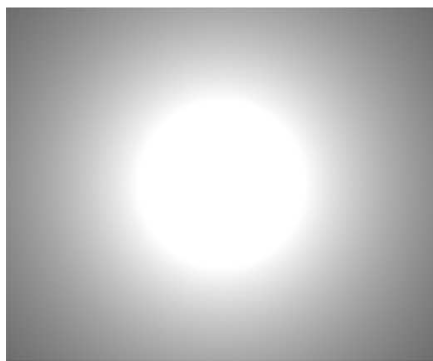

(e) Pixel efficiency for $\Delta=1$. (b) Macular image for $\Delta=2.5$.

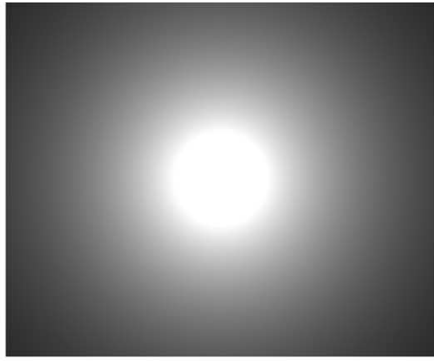

(f) Pixel efficiency for $\Delta=2.5$. (c) Macular image for $\Delta=10$.

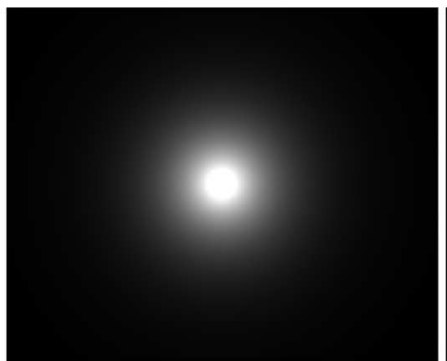

(g) Pixel efficiency for $\Delta=10$. (d) Macular image for $\Delta=150$.

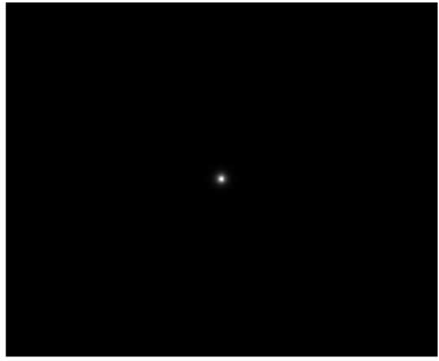

(h) Pixel efficiency for $\Delta=150$.

Fig. 6. $\Delta$-modified macular images and the $\Delta$-modified pixel efficiencies of every pixel of an image of resolution $352 \times 288$, for a display of pixel density $\Gamma_{1}=\Gamma_{2}=200$ PPI, $S$ at coordinates $(176,144)$, and viewing distance $D=15$ in, for four different values of $\Delta$. (a) Macular image for $\Delta=1$. (b) Macular image for $\Delta=2.5$. (c) Macular image for $\Delta=10$. (d) Macular image for $\Delta=150$. (e) Pixel efficiency for $\Delta=1$. (f) Pixel efficiency for $\Delta=2.5$. (g) Pixel efficiency for $\Delta=10$. (h) Pixel efficiency for $\Delta=150$.

photoreceptors layer, but also in subsequent layers of the human visual processing.

For example, according to [39], although the distribution of photoreceptors sets the upper limit on the range of spatial frequencies available to the retina, the distribution of ganglion cells sets the upper limit on the proportion of this information that is ultimately transmitted to the brain. More specifically, again according to [39]: comparison of ganglion cell topography with the visual field representation in V1 reveals similarities consistent with the idea that cortical magnification is proportional to ganglion cell density throughout the visual field; ganglion cell density decreases smoothly with eccentricity from about $1 \mathrm{~mm}$ radius of the foveal center; low convergence of foveal cones onto individual ganglion cells is an important mechanism for preserving high resolution at later stages of neural processing; and the number of cones per ganglion cell can increase smoothly with eccentricity from $1 \mathrm{~mm}$ radius of the foveal center ${ }^{2}$.

However, still according to [39], there is substantial individual variability in the number of ganglion cells, and substantial individual differences in the local number of cones per ganglion cell, whereas individual variability in the peripheral cone distribution is small. Then, since some of the fundamental pillars of visual acuity seem to vary substantially among individuals, for simplicity, we decided to model the aforementioned proportion of information that is ultimately transmitted to the brain by some power of a previously calculated monotonically decreasing function normalized by its peak value. Formally, the information reduction factor $g_{D, \Delta}\left[p_{1}, p_{2}\right]$ of a pixel centered at $P$ can be defined as:

$$
g_{D, \Delta}\left[p_{1}, p_{2}\right] \triangleq\left(\frac{q_{D}\left[p_{1}, p_{2}\right]}{q_{D}[0,0]}\right)^{\Delta}
$$

where $q_{D}\left[p_{1}, p_{2}\right]$ is given by (4).

\footnotetext{
${ }^{2}$ The number of cones per ganglion cell is a measure of potential convergence only.
}

Since such reduction does not change the retinotopic behavior of the images formed in the primary visual cortex, we believe foveated images are more pleasant and more naturally processed by the human brain than typical compressed images. With that in mind, we modify a few entities presented so far in order to allow visual perceptual loss. We start by the $\Delta$-modified number of cones per pixel $q_{D, \Delta}\left[p_{1}, p_{2}\right]$ of a pixel centered at $P$ that can be defined as:

$$
q_{D, \Delta}\left[p_{1}, p_{2}\right] \triangleq q_{D}\left[p_{1}, p_{2}\right] g_{D, \Delta}\left[p_{1}, p_{2}\right],
$$

where $q_{D}\left[p_{1}, p_{2}\right]$ is given by (4) and $g_{D, \Delta}\left[p_{1}, p_{2}\right]$ is given by (8).

This modified version of the number of cones per pixel just reduces its value pointwisely according to the information reduction factor $g_{D, \Delta}\left[p_{1}, p_{2}\right]$. Such change consequently impacts on the pixel efficiency, as the $\Delta$-modified pixel efficiency $e_{D, \Delta}\left[p_{1}, p_{2}\right]$ of a pixel centered at $P$ can be defined as:

$$
e_{D, \Delta}\left[p_{1}, p_{2}\right] \triangleq \begin{cases}1, & \text { if } q_{D, \Delta}\left[p_{1}, p_{2}\right] \geq 1 \\ q_{D, \Delta}\left[p_{1}, p_{2}\right], & \text { else, }\end{cases}
$$

where $q_{D, \Delta}\left[p_{1}, p_{2}\right]$ is given by (9).

Fig. 6 shows the $\Delta$-modified macular images and the $\Delta$-modified pixel efficiencies of every pixel, where the pixel efficiency of a pixel can vary from 0 (displayed as black) to 1 (displayed as white), of an image of resolution $352 \times 288$, for a display of pixel density $\Gamma_{1}=\Gamma_{2}=200$ PPI, $S$ at coordinates $(176,144)$, and viewing distance $D=15$ in, for four different values of $\Delta$.

When comparing Figs. 5 and 6, we can see that for a fixed value of $\Delta$ the macular images can vary from the whole image (Fig. 5(a)) to no image (Fig. 5(d)) as $D$ increases, whereas for a fixed value of $D$, the $\Delta$-modified macular images can vary from a macular image (Fig. 5(b)) to a single pixel (Fig. 6(d)) at $S$ as $\Delta$ increases.

Finally, the standard deviation of the new filter becomes $\sigma_{p_{1} p_{2}}=-0.38 \ln e_{D, \Delta}\left[p_{1}, p_{2}\right]$ for a pixel centered at $P$. Making a simple analysis of this new version of the filter we 
can see that: when $\Delta=0$ we have loss, however, it may be considered perceptually lossless; and when $\Delta>0$ we have visual perceptual loss. In conclusion, through the use of the lossy parameter $\Delta$, the proposed Gaussian foveation scheme permits controlling the strength of the foveation filtering, i.e., how much visual perceptual loss will be allowed.

\section{Comparison Between Foveation Filtering Methods}

The following material provides a comparison between the proposed method and a method introduced by Lee et al. in a series of work [15], [28]-[31].

\section{A. Model of Image Projection Onto the Retina}

Lee et al. calculates the eccentricity $e_{\mathbf{x}}$ (in degrees) at a point $\mathbf{x}=\left(x_{1}, x_{2}\right)$ in [27], [29] as:

$$
e_{\mathbf{x}}=\tan ^{-1}\left(\frac{i_{d} d_{\mathbf{x}}}{v_{d} i_{p}}\right)
$$

where $i_{p}$ is the image size (in pixels), $i_{d}$ is the image size (in units of length), $v_{d}$ is the distance from the viewer to the image (in the same units of length), and $d_{\mathbf{x}}$ is the distance (in pixels) from the foveation point in the image to a pixel at $\mathbf{x}$.

From the (11), one can see that apparently [27], [29] considers the pixel density the same for horizontal and vertical directions, whereas we consider that the pixel density can be different for horizontal and vertical directions. Furthermore, we can see that the projection model of Lee et al. can be considered a subset of our projection model if: $\Gamma_{1}=\Gamma_{2}=\left(i_{p} / i_{d}\right)$, $D=v_{d}$, and $d_{\mathbf{x}}=\sqrt{p_{1}^{2}+p_{2}^{2}}$. When such conditions hold, we have $\left(d_{D}\left[p_{1}, p_{2}\right] / 2 r\right) \equiv\left(\pi e_{\mathbf{x}} / 180\right)$, which shows that our model is more flexible regarding pixel density.

\section{B. Model of Cones Distribution}

The compared method uses a plot about the human visual detectable frequencies derived based on the photoreceptor density that can be seen in [27], [29]. A model for the mentioned plot is proposed, and is given by:

$$
f_{d_{\mathbf{x}}}=\frac{\gamma}{e_{\mathbf{x}}+\eta}
$$

where $e_{\mathbf{x}}$ is given by (11), and, according to [27], the parameters $\gamma=18$ and $\eta=0.2$ were chosen experimentally such that the human eye could not discriminate the foveated image from the original image for a given fixation point.

Considering [27], one could say the model given by (12) was fitted with the photoreceptor density by its peak. According to [38], this does not seem to be the best way to proceed, since the region of the retina with the highest variation on the number of cones across different subjects is the fovea, i.e., the region corresponding to the peak of the mentioned plot. This plays a major role in the modeling if we observe the high relative error between the curve based on the density ${ }^{3}$ and the curve based on the model found in [27], [29]. Regarding our modeling, one can

${ }^{3}$ Considering that such density refers to cones only. say that the relative error between the distributions found in [38] and the model itself is smaller.

Regarding both methods, those models are used to determine the parameters of the filters in different ways. The compared method uses local bandwidth in cycles per pixel, while we use cones per pixel. Yet, we will show that both approaches share the same idea.

The compared method uses a conversion factor $\beta_{\mathbf{x}}$ [27], to convert $f_{d_{\mathbf{x}}}$ (in cycles/degree) into $f_{p_{\mathbf{n}}}$ (in cycles/pixels). It supposes each pixel forms a square with the length of each side $\epsilon=\left(i_{d} / i_{p}\right)$. Then, $\beta_{\mathbf{x}}$ in its corrected is approximately ${ }^{4}$ :

$$
\beta_{\mathbf{x}}=\tan ^{-1}\left(\frac{\epsilon}{2 v_{d}}+\frac{\epsilon d_{\mathbf{x}}}{v_{d}}\right)-\tan ^{-1}\left(\frac{-\epsilon}{2 v_{d}}+\frac{\epsilon d_{\mathbf{x}}}{v_{d}}\right) .
$$

The conversion is given by: $f_{p_{\mathbf{n}}}=\beta_{\mathbf{x}} f_{d_{\mathbf{x}}}$. Then, $f_{p_{\mathbf{n}}}$ is thresholded at the maximum normalized frequency 0.5 and at a minimum bandwidth 0.07 . A plot of the resulting local bandwidth (in cycles/pixels) can be found in [29].

Thresholding $f_{p_{\mathbf{n}}}$ by a minimum bandwidth does not seem to be the best approach for many different applications. When a subject is far enough from a display, the resulting local bandwidth at some pixels can be smaller than a given threshold value.

On the other hand, thresholding $f_{p_{\mathbf{n}}}$ at the maximum normalized frequency seems to be very appropriate. When Lee et al. argue that $f_{p_{\mathbf{n}}}$ should be thresholded at the maximum normalized frequency 0.5 , we can assume they are actually arguing that in order to "perceive" 1 cycle we would need two samples, i.e., 2 photoreceptors, which is the same as arguing that every photoreceptor can only "perceive" 0.5 cycles. They define the local bandwidth for every pixel, while we define the number of samples for every pixel, which, according to the Sampling Theorem, must be at least two times the bandwidth of the signal. In a nutshell, we have that $2 \beta_{\mathbf{x}} f_{d_{\mathbf{x}}}$ should be equal to $q_{D}\left[p_{1}, p_{2}\right]$.

\section{Foveation Filters}

The compared method uses an approximation $h(i)$ of an ideal lowpass filter $h^{*}(i)$, while we use an approximation $h_{m_{1} m_{2}}\left[n_{1}, n_{2}\right]$ of an ideal Gaussian filter. The design in [27], [29] aims to minimize the total energy of the error signal $e$ between $h^{*}(i)$ and $h(i)$ given by:

$$
e=\sum_{i=-\infty}^{\infty}\left[h^{*}(i)-h(i)\right]^{2} \text {. }
$$

In order to do so, $h(i)$ must be:

$$
h(i)= \begin{cases}\frac{\omega_{c}}{\pi} \operatorname{sinc}\left(\omega_{c} i\right), & \text { if }-\frac{N}{2} \leq i \leq \frac{N}{2} \\ 0, & \text { else, }\end{cases}
$$

where $\omega_{c}=2 \pi f_{p_{\mathbf{n}}}$ and $N$ is the length of filter minus 1 .

Also, [27], [29] calculates the error ratio $\tau$ relative to the total energy as:

$$
\tau=\frac{e \pi}{\omega_{c}}
$$

\footnotetext{
${ }^{4} \beta_{\mathbf{x}}$ resembles $A_{D}\left[p_{1}, p_{2}\right]$ in a sense that they are both related to the size of a pixel.
} 
TABLE I

Perceptual Efficiency of a Display for Phones

\begin{tabular}{|c|c|c|c|}
\hline & $\begin{array}{c}\text { Near } \\
5 \%\end{array}$ & $\begin{array}{c}\text { Typical } \\
10 "\end{array}$ & $\begin{array}{c}\text { Far } \\
15 \%\end{array}$ \\
\hline Samsung Galaxy SIII & $99 \%$ & $59 \%$ & $33 \%$ \\
\hline Apple iPhone 5 & $99 \%$ & $58 \%$ & $32 \%$ \\
\hline LG Optimus G LS970 & $98 \%$ & $55 \%$ & $31 \%$ \\
\hline Typical (480 × 800, 260 PPI) & $100 \%$ & $87 \%$ & $53 \%$ \\
\hline
\end{tabular}

TABLE II

Perceptual EFficiency of a Display for TABlets

\begin{tabular}{|c|c|c|c|}
\hline & $\begin{array}{c}\text { Near } \\
10 "\end{array}$ & $\begin{array}{c}\text { Typical } \\
15 "\end{array}$ & $\begin{array}{c}\text { Far } \\
20 "\end{array}$ \\
\hline Apple iPad Retina display & $48 \%$ & $29 \%$ & $19 \%$ \\
\hline Amazon Kindle Fire HD 8.9" & $55 \%$ & $33 \%$ & $22 \%$ \\
\hline Samsung Galaxy Tab 10.1 & $100 \%$ & $78 \%$ & $59 \%$ \\
\hline Typical (1920 × 1200, 200 PPI) & $71 \%$ & $45 \%$ & $31 \%$ \\
\hline
\end{tabular}

As we can see, for a given maximum error ratio $\tau$, if $\omega_{c}$ decreases, then $e$ must decrease as well, which implies an increase on the length of the filter. A plot showing the number of filter taps according to cutoff frequency for different values of $\tau$ can be found in [29]. Also, since $\omega_{c}=2 \pi f_{p_{\mathbf{n}}}$, we can see that the length of the filter will vary according to the pixel location.

In order to implement the filters in a fast way, the compared method uses two different approaches: one consisting on designing separable even symmetric filters and the other on designing circularly symmetric filters. In both approaches Lee et al. use Hamming windows to smooth the frequency response of the filters. Ideally, such windowing technique would require a new analysis on the filter approximation. We do not face this problem since we do not use such windowing technique in our proposed method.

Also, the filtering scheme in [29] uses a filter bank with limited number (less than 25) of different filters for a given value of $\tau$, while our method does not have a limit on the number of filters, and can also be limited if desired. Moreover, since we are using Gaussian filters, our approach for the filter design is separable even symmetric and circularly symmetric at the same time, while the compared approach can only be one or the other.

Finally, a crucial point regarding the comparison between methods is the use of a lossy parameter. The filtering scheme presented by Lee et al. does not consider the use of such artifice, while our method permits the use of the lossy parameter $\Delta$ to allow visual perceptual loss that might be necessary due to bandwidth limitations on transmissions.

\section{RESULTS}

Tables I-IV show the perceptual efficiency of a display for different kinds of devices. For every table we show the perceptual efficiency of a display of three current top seller devices in addition to a typical device, i.e., a display whose specifications can be considered typical among a comprehensive list of devices $^{5}$. All displays are analyzed under three different viewing distances: near, typical, and far. For phones, tablets, and note-

\footnotetext{
${ }^{5}$ http://en.wikipedia.org/wiki/List_of_displays_by_pixel_density
}

TABLE III

PERCEPTUAL EFFICIENCY OF A DisPlay FOR NOTEBOOKS

\begin{tabular}{|c|c|c|c|}
\hline & $\begin{array}{c}\text { Near } \\
10 "\end{array}$ & $\begin{array}{c}\text { Typical } \\
20 "\end{array}$ & $\begin{array}{c}\text { Far } \\
30 "\end{array}$ \\
\hline Apple MacBook Pro 13-inch Retina display & $53 \%$ & $22 \%$ & $12 \%$ \\
\hline Sony Vaio Z Series & $85 \%$ & $40 \%$ & $23 \%$ \\
\hline Toshiba Portégé & $100 \%$ & $74 \%$ & $47 \%$ \\
\hline Typical (1366 $\times 768,130$ PPI) & $100 \%$ & $67 \%$ & $41 \%$ \\
\hline
\end{tabular}

TABLE IV

PERCEPTUAL EFFICIENCY OF A DisPlay FOR TELEVISIONS

\begin{tabular}{|c|c|c|c|}
\hline & $\begin{array}{c}\text { Near } \\
50 "\end{array}$ & $\begin{array}{c}\text { Typical } \\
100 "\end{array}$ & $\begin{array}{c}\text { Far } \\
150 "\end{array}$ \\
\hline TV 32" & $31 \%$ & $12 \%$ & $6 \%$ \\
\hline TV 42" & $55 \%$ & $22 \%$ & $12 \%$ \\
\hline TV 50" & $65 \%$ & $28 \%$ & $16 \%$ \\
\hline Typical (1920 × 1280, 45 PPI) & $64 \%$ & $27 \%$ & $15 \%$ \\
\hline
\end{tabular}

books, the typical viewing distance was extracted from information related to top seller devices ${ }^{6}$. For TVs we used an approximation of the average viewing distance reported in [44]. For near and far viewing distances we used appropriate common sense measurements.

As we can see from Tables I-IV, values for the perceptual efficiency of a display can be considered low for typical viewing distances, and even lower for far viewing distances. Specifically, results show that we can achieve data reduction ratios up to approximately $3: 1,9: 1$, and 16:1 with no visual perceptual loss under a near, typical, and far viewing distances, respectively.

Fig. 7 shows how the perceptual efficiency of a display varies according to its parameters. Basically, according to (6), the perceptual efficiency of a display can vary according to the pixel densities $\Gamma_{1}$ and $\Gamma_{2}$, the image size $N_{1} \times N_{2}$, and the viewing distance $D$. However, it can be hard to analyze how the proposed limit changes in terms of five parameters. So, for simplicity, we display the results according to (7), i.e., considering only three parameters: $\Gamma=\Gamma_{1}=\Gamma_{2}, N=N_{1}=N_{2}$ and $D$. Since we have three parameters to vary, we need three 3-D plots to display the results. For each plot, we keep one of the parameters constant, and vary the other two, so we can see how $E$ changes in terms of its parameters. In Fig. 7(a), we conveniently kept $D=15$ in since it can be considered to be the typical viewing distance for tablets (Table II) and it can also be considered to be an approximation to the standard reading distance [45]. In Fig. 7(b), we held $N=512$ since it can be considered one of the most common sizes for images [46]. Finally, in Fig. 7(c), we made $\Gamma=200$ PPI since it can be considered the pixel density of a typical tablet (Table II).

Results from Fig. 7 show how $E$ drops for big enough values of its parameters, since the proposed limit is not monotonically decreasing in terms of $D$, nor it is in terms of $\Gamma$. Also, we can see that, in general, $E$ falls slower in terms of $N$ than in terms of its other two parameters. In fact, it turns out that $\Gamma$ and $D$ play the same role in the evaluation of the perceptual efficiency of a display. According to (1), if $\Gamma=\Gamma_{1}=\Gamma_{2}$, then:

$$
d_{D}\left[p_{1}, p_{2}\right]=2 r \arctan \left(\frac{\sqrt{p_{1}^{2}+p_{2}^{2}}}{D \Gamma}\right) .
$$

${ }^{6} \mathrm{http}: / /$ en.wikipedia.org/wiki/Retina_Display 


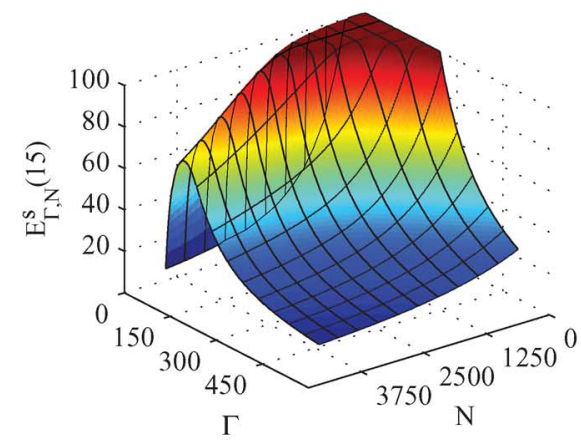

(a) $E$ for $D=15$.

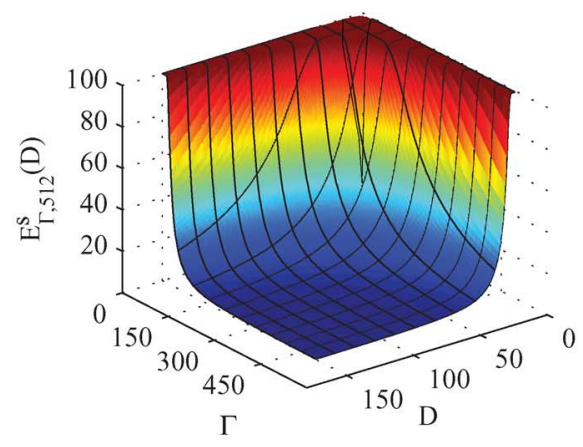

(b) $E$ for $N=N_{1}=N_{2}=512$.

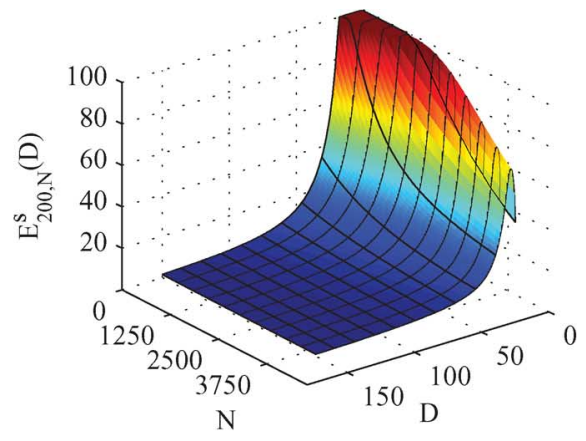

(c) $E$ for $\Gamma=\Gamma_{1}=\Gamma_{2}=200$.

Fig. 7. Perceptual efficiency of a display for different values of $\Gamma=\Gamma_{1}=\Gamma_{2}, N=N_{1}=N_{2}$, and $D$. (a) $E$ for $D=15$. (b) $E$ for $N=N_{1}=N_{2}=512$. (c) $E$ for $\Gamma=\Gamma_{1}=\Gamma_{2}=200$.

Also, according to (2), if $\Gamma=\Gamma_{1}=\Gamma_{2}$, then:

$$
\begin{aligned}
A_{D}\left[p_{1}, p_{2}\right] \approx & 4 r^{2}\left[\arctan \left(\frac{p_{1}+0.5}{D \Gamma}\right)-\arctan \left(\frac{p_{1}-0.5}{D \Gamma}\right)\right] \\
& \times\left[\arctan \left(\frac{p_{2}+0.5}{D \Gamma}\right)-\arctan \left(\frac{p_{2}-0.5}{D \Gamma}\right)\right] .
\end{aligned}
$$

This means that the values of $D$ and $\Gamma$ are completely interchangeable. Moreover, in this case, $d_{D}\left[p_{1}, p_{2}\right]$ and $A_{D}\left[p_{1}, p_{2}\right]$ depend only on the product $D \Gamma$. Such remark also impacts on the results of the proposed Gaussian foveation scheme.

Fig. 8 shows compression results for a frame of the CIF sequence foreman. All compressed frames were encoded using the H.264/AVC reference software JM version 18.5. Except for the quantization pass $Q P$, they were all encoded using the same configuration?. We wanted to verify how much foveation filtering could possibly improve the perceptual quality of an uncompressed frame, so we first encoded it using $Q P=50$ with no foveation scheme. Then, we performed foveation filtering in the original frame and encoded it. In order to provide a better comparison between methods, we will assume the conditions in Section VI-A hold, i.e., $\Gamma_{1}=\Gamma_{2}=\left(i_{p} / i_{d}\right), D=v_{d}$, and $d_{\mathbf{x}}=\sqrt{p_{1}^{2}+p_{2}^{2}}$. So, for the method developed by Lee et al. [29] we made $D \Gamma=3000$. For the proposed method we also made $D \Gamma=3000$ and chose appropriate values for $\Delta$ and $Q P$. Results display the 10th frames. The point $S$ for the 10th frame is marked with a green cross 8 in Fig. 8(a). For convenience, we made $\Gamma=200$ PPI so that $D=15$ in becomes the actual viewing distance for the reader.

As we can see from Fig. 8, the result regarding the proposed preprocessing method can clearly enhance the perceptual quality of an image (if we consider that the region of interest in such image is centered at $S$ ) under the bit rate restriction. In Fig. 8(b) and 8(c) we can clearly see blocking artifacts around $S$, whereas in Fig. $8(\mathrm{~d})$ we can see a much smoother image around the same point. Such results indicate that the last question of our problem statement can be answered positively. Moreover, the result regarding the proposed method can also be considered better than the one provided by Lee et al. [29].

\footnotetext{
${ }^{7}$ The configuration can be found in the file encoder.cfg (provided by the reference software) with the following changes: FramesToBeEncoded $=15 ;$ SourceWidth $=352 ;$ SourceHeigh $t=288 ;$ LevelIDC $=42$; IntraPeriod $=15 ;$ QPISlice $=Q P ;$ QPPSlice $=Q P ;$ SearchRange $=$ 16 ; NumberReferenceFrames $=4$; NumberBFrames $=2 ;$ QPBSlice $=$ $Q P$; and HierarchicalCoding $=0$.

${ }^{8} S$ is at coordinates $(238,200)$.
}

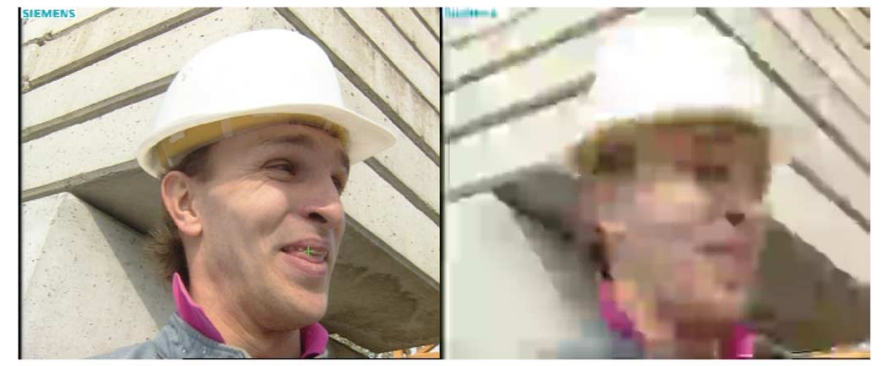

(a) Original.

(b) At $0.015 \mathrm{bpp}(Q P=50)$.

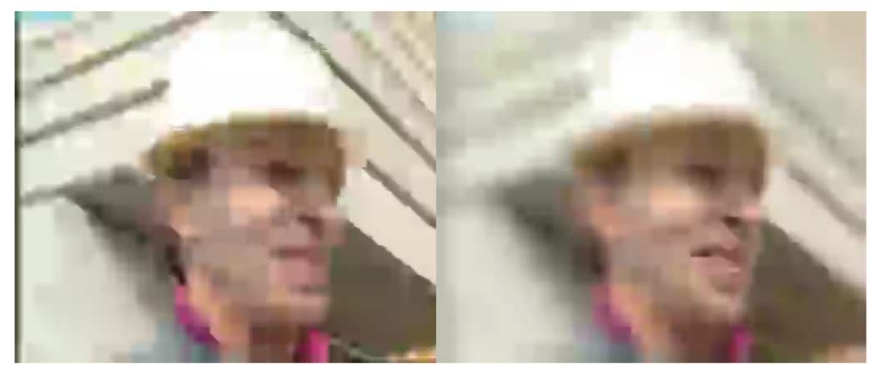

(c) Preprocessed by Lee et al. [29] (d) Preprocessed by proposed method at $0.016 \mathrm{bpp}(Q P=50)$

$(\Delta=40)$ at $0.015 \mathrm{bpp}(Q P=46)$.

Fig. 8. Compression results for the 10th frame of the CIF sequence foreman, for $\Gamma=\Gamma_{1}=\Gamma_{2}=200$ PPI, and $D=15$ in. (a) Original. (b) At $0.015 \mathrm{bpp}$ $(Q P=50)$. (c) Preprocessed by Lee et al. [29] at 0.016 bpp $(Q P=50)$. (d) Preprocessed by proposed method $(\Delta=40)$ at $0.015 \operatorname{bpp}(Q P=46)$.

Fig. 9 shows compression results for a frame of the $720 \mathrm{p}$ sequence parkrun. Again, All compressed frames were all encoded using the H.264/AVC reference software JM version 18.5 , and, except for the quantization pass $Q P$, they were encoded using the same configuration ${ }^{9}$. Once again, we wanted to verify how much foveation filtering could possibly improve the perceptual quality of an uncompressed frame. In order to do so, we proceeded like before. Results display the 10th frames. Since the displayed frames have much more pixels than the ones shown in Fig. 8, we also displayed magnified macular images of such frames in order to facilitate the visualization of the results. The point $S$ for the 10th frame is located at the center of its macular image ${ }^{10}$. For convenience, we now made $\Gamma=600$ PPI so that $D=5$ in becomes the actual viewing

\footnotetext{
${ }^{9}$ The only differences between this configuration and the previous one are the following: FrameRate $=50$; SourceWidth $=1280$; and SourceHeight $=$ 720 .

${ }^{10} \mathrm{~S}$ is at coordinates $(616,396)$.
} 


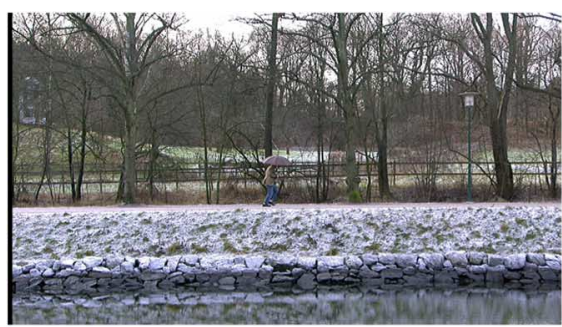

(a) Original.

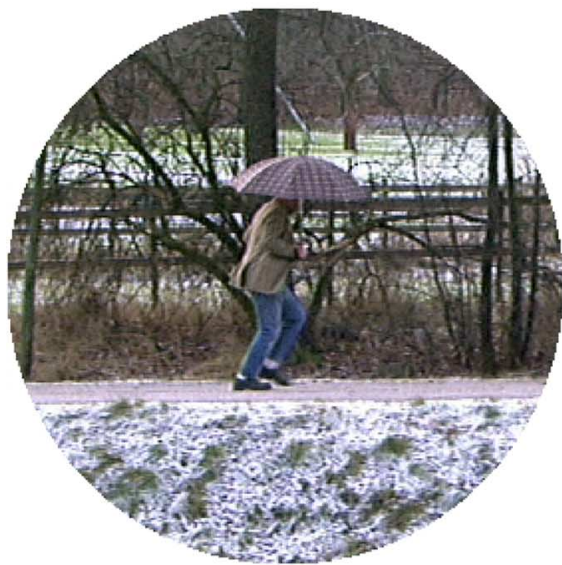

(d) Magnified macular image of the frame in Fig. 9(a).

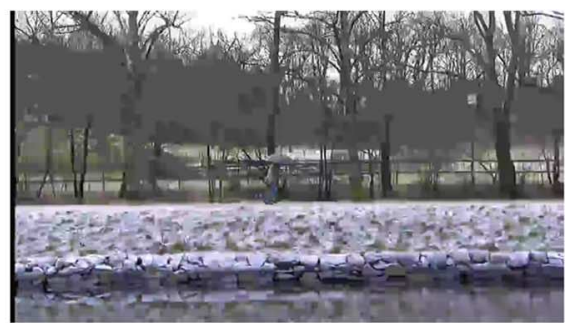

(b) At 0.019 bpp $(Q P=50)$.

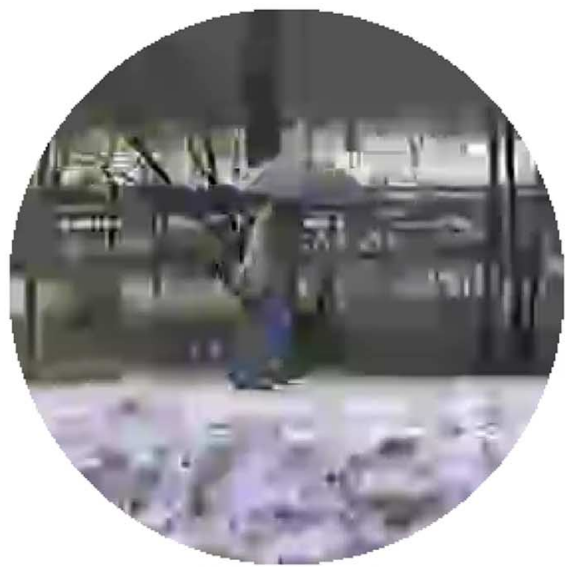

(e) Magnified macular image of the frame in Fig. 9 (b).

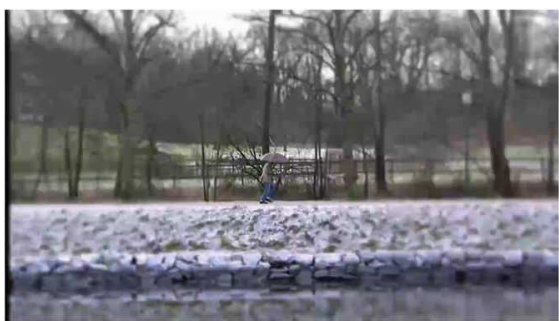

(c) Preprocessed by proposed method $(\Delta=7)$ at 0.014 bpp $(Q P=42)$.

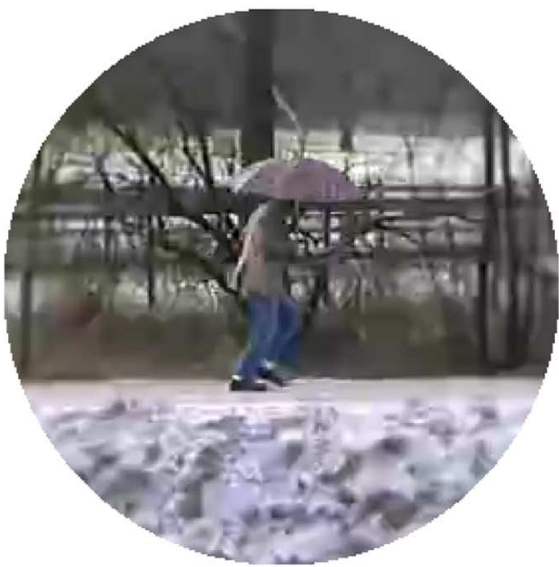

(f) Magnified macular image of the frame in Fig. 9(c).

Fig. 9. Compression results for the 10th frame of the 720p sequence parkrun, for $\Gamma=\Gamma_{1}=\Gamma_{2}=600$ PPI, and $D=5$ in. (a) Original. (b) At 0.019 bpp $(Q P=50)$. (c) Preprocessed by proposed method $(\Delta=7)$ at 0.014 bpp $(Q P=42)$. (d) Magnified macular image of the frame in Fig. 9(a). (e) Magnified macular image of the frame in Fig. 9(b);. (f) Magnified macular image of the frame in Fig. 9(c).

distance for the reader (note that the product $D \Gamma$ is still the same).

Again, as we can see from Fig. 9, the result regarding the proposed preprocessing method can clearly enhance the perceptual quality of an image (if we consider that the region of interest in such image is centered at $S$ ) under the bit rate restriction. Although we can see a substantial amount of visual perceptual loss in Fig. 9(b) and 9(c) when compared to Fig. 9(a), when we look at the magnified macular images in Fig. 9(e) and 9(f) we can clearly see that the latter looks much better than the former, implying that the perceptual quality of the frame in Fig. 9(c) can be considered better than the perceptual quality of the frame in Fig. 9(b). Such results corroborate the claim that the last question of our problem statement can be answered positively. Also, it becomes clear that the enhancement of the perceptual quality is highly dependent on the source complexity.

\section{A. Subjective Experiments}

In order to properly test the proposed method, we performed extensive viewing tests with human subjects. Ten subjects (five males and five females) participated in this study. In such tests, each subject was asked to grade each image $x$ a few times in order to assess its perceptual quality $Q(x)$. In order to provide enough variety of content, the 10th frame of fourteen different $1080 \mathrm{p}$ sequences $s_{1}, s_{2}, \ldots, s_{14}$ were preprocessed by two different methods: Lee et al. [29] $L(\cdot)$; and the proposed method $T_{\Delta}(\cdot)$. Regarding each $s_{i}$, a total of twenty-three frames became available upon such procedure: one original frame $s_{i}$; one frame preprocessed by Lee et al. [29] $L\left(s_{i}\right)$; and twenty-one frames preprocessed by the proposed method $T_{0}\left(s_{i}\right), T_{1}\left(s_{i}\right), \ldots, T_{20}\left(s_{i}\right)$. Now, each one of such twenty-three frames were compressed using the H.264/AVC reference software JM version 18.5 using the same configuration ${ }^{11} C_{Q P}(\cdot)$, for $Q P=36,37, \ldots, 50$. For each frame $s_{i}$, a total of 345 compressed frames became available upon such procedure: fifteen non-preprocessed compressed frames $C_{36}\left(s_{i}\right), C_{37}\left(s_{i}\right), \ldots, C_{50}\left(s_{i}\right)$; fifteen compressed frames preprocessed by Lee et al. [29] $C_{36}\left(L\left(s_{i}\right)\right), C_{37}\left(L\left(s_{i}\right)\right), \ldots, C_{50}\left(L\left(s_{i}\right)\right) ; \quad$ and 315 compressed frames preprocessed by the proposed method $C_{36}\left(T_{0}\left(s_{i}\right)\right), C_{37}\left(T_{0}\left(s_{i}\right)\right), \ldots, C_{50}\left(T_{0}\left(s_{i}\right)\right) ; C_{36}\left(T_{1}\left(s_{i}\right)\right), C_{37}$ $\left(T_{1}\left(s_{i}\right)\right), \ldots, C_{50}\left(T_{1}\left(s_{i}\right)\right) ; \ldots ; C_{36}\left(T_{20}\left(s_{i}\right)\right), C_{37}\left(T_{20}\left(s_{i}\right)\right), \ldots$, $C_{50}\left(T_{20}\left(s_{i}\right)\right)$. Now, regarding each original frame $s_{i}$, and considering that each of the compressed frames are associated to a bit rate $B(\cdot)$, the following thirteen frames were selected to participate in the subjective experiments:

- $s_{i}$;

- $T_{0}\left(s_{i}\right)$

- $C_{50}\left(s_{i}\right)$

- Four frames from $C_{36}\left(s_{i}\right), C_{37}\left(s_{i}\right), \ldots, C_{49}\left(s_{i}\right)$;

- $C_{Q P^{*}}\left(L\left(s_{i}\right)\right)$, where $Q P_{i}^{*}$ is the smallest possible such that $B\left(C_{Q P^{*}}\left(L\left(s_{i}\right)\right)\right)<B\left(C_{50}\left(s_{i}\right)\right)$;

- For each of five different values of $\Delta$, i.e., $\Delta=\Delta_{i, j}$ for $j=1,2, \ldots 5$, a frame $C_{Q P_{i, j}}\left(T_{\Delta_{i, j}}\left(s_{i}\right)\right)$, where $Q P_{i, j}$ is the smallest possible such that $B\left(C_{Q P_{i, j}}\left(T_{\Delta_{i, j}}\left(s_{i}\right)\right)\right)<$ $B\left(C_{Q P_{i}^{*}}\left(L\left(s_{i}\right)\right)\right)$.

${ }^{11}$ The only differences between this configuration and the previous one are the following: FrameRate $=25$ (for frames $\left.s_{3}, s_{4}, s_{9}, s_{14}\right)$; SourceWidth $=$ 1920; and SourceHeight $=1080$. 


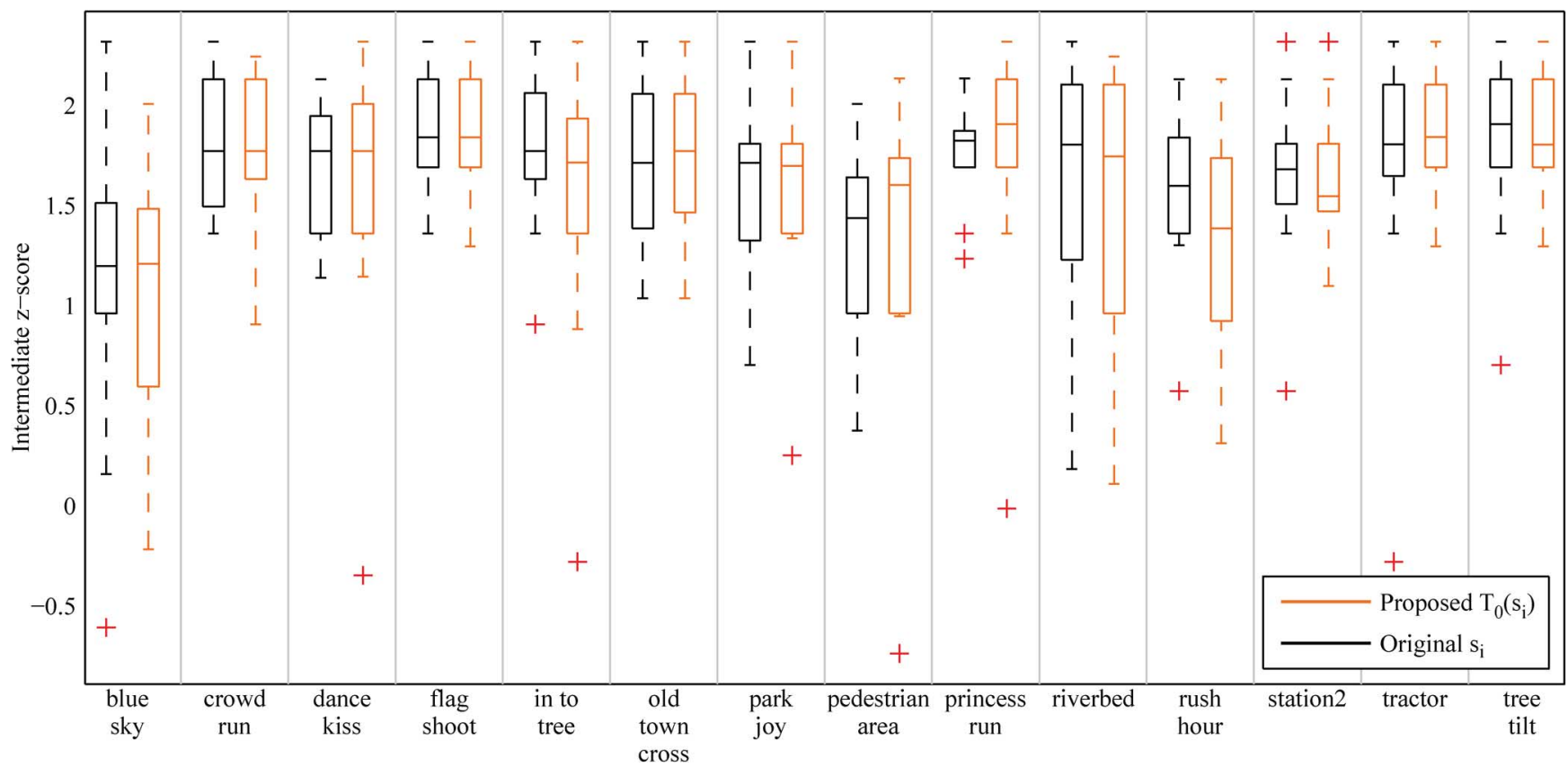

Fig. 10. Box plot results for intermediate z-scores of the frames $s_{i}$ and the preprocessed frames $T_{0}\left(s_{i}\right)$, for $i=1,2, \ldots, 14$.

TABLE V

MOS Results and Their 95\% CONFidence InTERVAls For the Non-Preprocessed Compressed Frames $C_{50}\left(s_{i}\right)$, the Compressed Frames Preprocessed by Lee et al. [29] $C_{Q P^{*}}\left(L\left(s_{i}\right)\right)$, AND one of the Compressed Frames Preprocessed by the Proposed Method $C_{Q P_{i, j}}\left(T_{\Delta_{i, j}}\left(s_{i}\right)\right)$, For $i=1,2, \ldots, 14$

\begin{tabular}{|c|c|c|c|c|c|c|c|c|c|c|c|c|c|c|}
\hline & $\begin{array}{l}\text { blue } \\
\text { sky }\end{array}$ & $\begin{array}{l}\text { crowd } \\
\text { run }\end{array}$ & $\begin{array}{c}\text { dance } \\
\text { kiss }\end{array}$ & $\begin{array}{l}\text { flag } \\
\text { shoot }\end{array}$ & $\begin{array}{l}\text { in to } \\
\text { tree }\end{array}$ & $\begin{array}{c}\text { old town } \\
\text { cross }\end{array}$ & $\begin{array}{l}\text { park } \\
\text { joy }\end{array}$ & $\begin{array}{c}\text { pedestrian } \\
\text { area }\end{array}$ & $\begin{array}{l}\text { princess } \\
\text { run }\end{array}$ & riverbed & $\begin{array}{l}\text { rush } \\
\text { hour }\end{array}$ & station 2 & tractor & $\begin{array}{l}\text { tree } \\
\text { tilt }\end{array}$ \\
\hline Non-preprocessed & $\begin{array}{c}1.88 \\
\pm 0.48\end{array}$ & $\begin{array}{c}2.87 \\
\pm 0.53\end{array}$ & $\begin{array}{r}1.80 \\
\pm 0.57\end{array}$ & $\begin{array}{c}2.29 \\
\pm 0.35\end{array}$ & $\begin{array}{r}2.55 \\
\pm 0.51\end{array}$ & $\begin{array}{c}2.24 \\
\pm 0.36\end{array}$ & $\begin{array}{c}1.61 \\
\pm 0.32\end{array}$ & $\begin{array}{c}1.48 \\
\pm 0.32\end{array}$ & $\begin{array}{c}1.78 \\
\pm 0.42\end{array}$ & $\begin{array}{c}1.80 \\
\pm 0.46\end{array}$ & $\begin{array}{c}1.93 \\
\pm 0.40\end{array}$ & $\begin{array}{c}1.58 \\
\pm 0.66\end{array}$ & $\begin{array}{c}2.45 \\
\pm 0.55\end{array}$ & $\begin{array}{c}2.05 \\
\pm 0.42\end{array}$ \\
\hline Lee et al. [29] & $\begin{array}{r}2.55 \\
\pm 0.57\end{array}$ & $\begin{array}{r}2.86 \\
\pm 0.48\end{array}$ & $\begin{array}{r}1.75 \\
+0.49\end{array}$ & $\begin{array}{r}2.52 \\
\pm 0.50\end{array}$ & $\begin{array}{r}\mathbf{2 . 7 8} \\
\pm 0.50\end{array}$ & $\begin{array}{c}2.00 \\
\pm 0.42\end{array}$ & $\begin{array}{c}2.24 \\
\pm 0.30\end{array}$ & $\begin{array}{c}1.59 \\
\pm 0.31\end{array}$ & $\begin{array}{c}2.23 \\
\pm 0.33\end{array}$ & $\begin{array}{r}1.88 \\
\pm 0.66\end{array}$ & $\begin{array}{c}2.11 \\
\pm 0.53\end{array}$ & $\begin{array}{r}1.48 \\
\pm 0.28\end{array}$ & $\begin{array}{r}2.30 \\
\pm 0.55\end{array}$ & $\begin{array}{r}2.43 \\
\pm 0.55\end{array}$ \\
\hline Proposed & $\begin{array}{c}2.89 \\
\pm 0.53\end{array}$ & $\begin{array}{c}\mathbf{3 . 0 0} \\
\pm 0.45\end{array}$ & $\begin{array}{c}2.02 \\
\pm 0.50\end{array}$ & $\begin{array}{c}2.68 \\
\pm 0.43\end{array}$ & $\begin{array}{c}2.76 \\
\pm 0.47\end{array}$ & $\begin{array}{c}2.54 \\
\pm 0.33\end{array}$ & $\begin{array}{c}2.70 \\
\pm 0.31\end{array}$ & $\begin{array}{c}\mathbf{1 . 8 8} \\
\pm 0.73\end{array}$ & $\begin{array}{c}2.67 \\
\pm 0.56\end{array}$ & $\begin{array}{c}\mathbf{1 . 9 6} \\
\pm 0.73\end{array}$ & $\begin{array}{c}2.13 \\
\pm 0.40\end{array}$ & $\begin{array}{r}\mathbf{1 . 9 6} \\
\pm 0.59\end{array}$ & $\begin{array}{c}\mathbf{2 . 5 6} \\
\pm 0.37\end{array}$ & $\begin{array}{c}\mathbf{2 . 6 0} \\
\pm 0.42 \\
\end{array}$ \\
\hline
\end{tabular}

Regarding the subjective test itself, we designed a software using the following algorithm for each subject:

1) Randomize the order of the frames;

2) Show a gray frame with a fixation point for $1250 \mathrm{~ms}$;

3) Show a 10th frame for $300 \mathrm{~ms}$;

4) Show a gray frame asking the subject to press a key to judge the image quality. The possible grades are $1,2, \ldots$, 9 , where 1 is poor and 9 is undistorted ${ }^{12}$;

5) Show a gray frame for $750 \mathrm{~ms}$;

6) Repeat steps $2-5$ one-hundred and eighty-one more times such that each one of the 182 selected frames receive grades;

7) Show a gray frame saying the subject can take a break;

8) Repeat steps 1-7 two more times, just to get a reliability measure.

Fig. 10 shows subjective results for the original frames $s_{i}$ and the preprocessed frames $T_{0}\left(s_{i}\right)$, for $i=1,2, \ldots, 14$. For each subject, we discarded the first block of trials, i.e., the first 182 out of 546 grades. Then we calculated the z-score for each one of the remaining 364 trials. Finally we averaged the two $z$-scores of each image, so that we generate an intermediate

\footnotetext{
${ }^{12} \mathrm{We}$ did not label the values between 1 and 9 since people use the scale differently, and labeling does not help. When we analyzed their ratings, we standardized the ratings to account for individual differences in how people use the scale.
}

z-score per image per subject. As an important note, different people can use different ranges of the scale (e.g., 1-9, 4-8, 1-5, $6-9)$. By standardizing each subject's ratings, we put them onto the same scale before comparing different methods. If we do not do that, then ratings from someone who uses the full scale will be weighted more than those from someone who does not. Normalizing reduces the noise from differences in how people use the scale to our estimates of the relative quality of the different methods.

As we can see from Fig. 10, the intermediate z-scores of the frames $s_{i}$ and $T_{0}\left(s_{i}\right)$ are fairly close to each other for each $i$, especially if we consider that they were displayed together with all the other ones in completely random orders to the subjects. We believe such results give enough psychological evidence to answer positively the first question of our problem statement, i.e., it is possible to preprocess an image $x$ yielding an image $y$ so that $Q(y)=Q(x)$.

Table $\mathrm{V}$ shows subjective results for the non-preprocessed compressed frames $C_{50}\left(s_{i}\right)$, the compressed frames preprocessed by Lee et al. [29] $C_{Q P^{*}}\left(L\left(s_{i}\right)\right)$, and one of the compressed frames preprocessed by the proposed method $C_{Q P_{i, j}}\left(T_{\Delta_{i, j}}\left(s_{i}\right)\right)$, for $i=1,2, \ldots, 14$. In order to generate the results, we proceed like before. Then we calculated a mean across all the subjects for each compressed image. Finally, we scale the mean intermediate $\mathrm{z}$-scores to $1-5$ range so that 


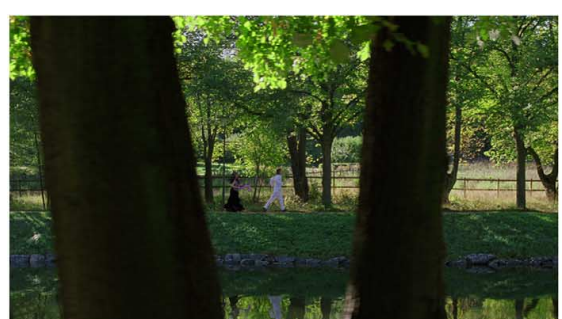

(a) Original.

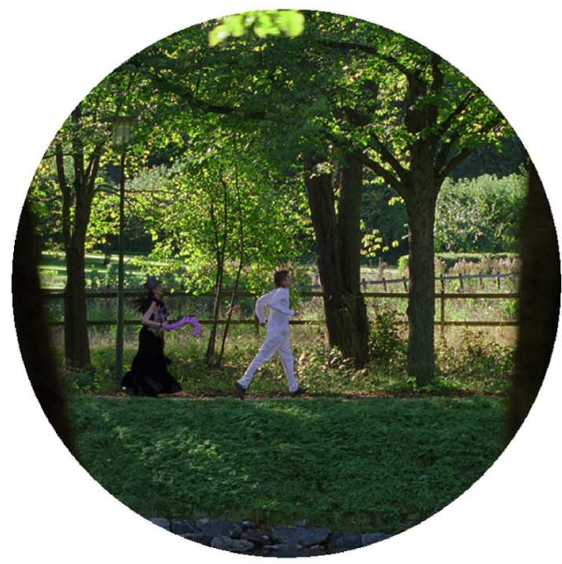

(d) Magnified macular image of the frame in Fig. 11(a).

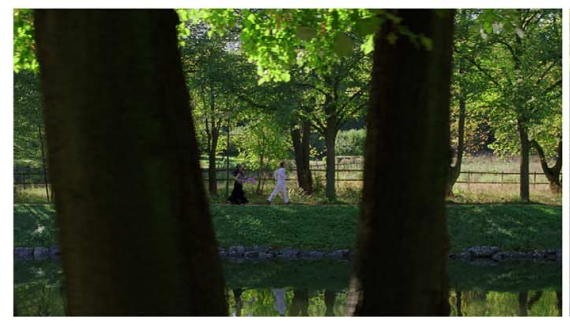

(g) Proposed method $(\Delta=0)$.

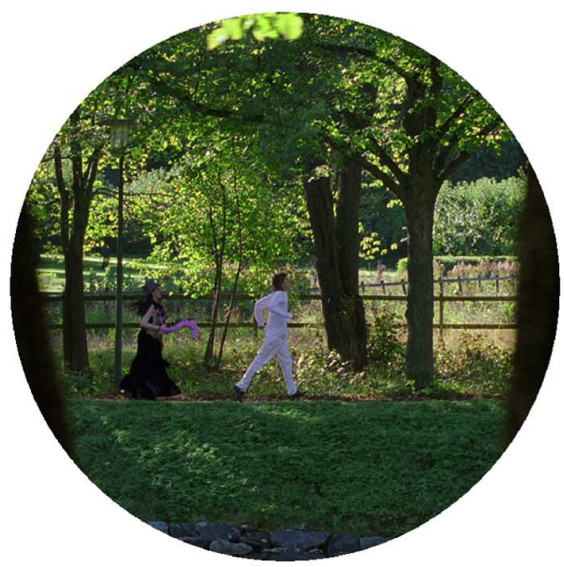

(j) Magnified macular image of the frame in Fig. 11(g).
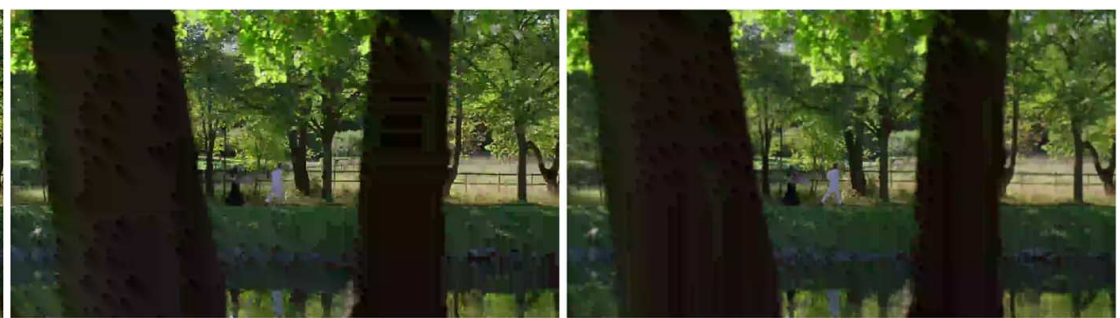

(b) At 0.029 bpp $(Q P=50), \operatorname{MOS}=1.61$.

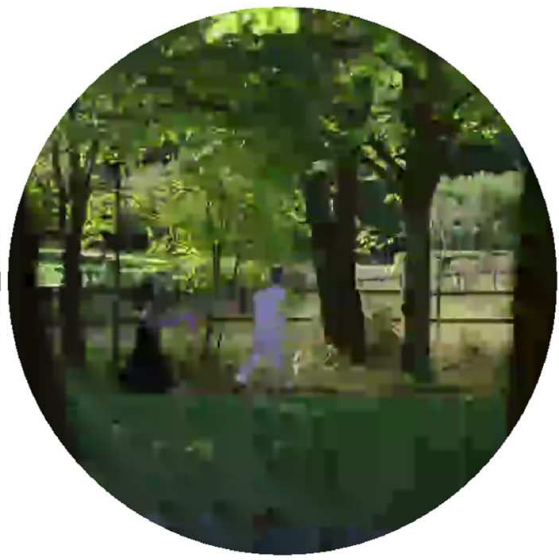

(e) Magnified macular image of the frame in Fig. 11(b).

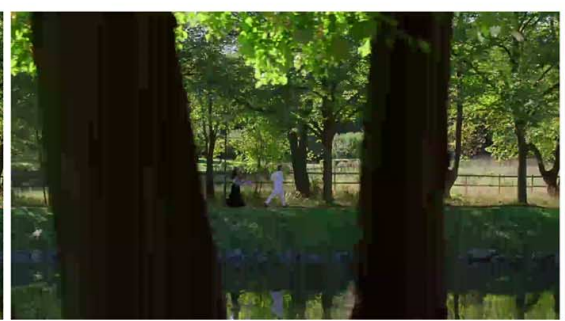

(h) At $0.049 \mathrm{bpp}(Q P=47), \operatorname{MOS}=2.42$.

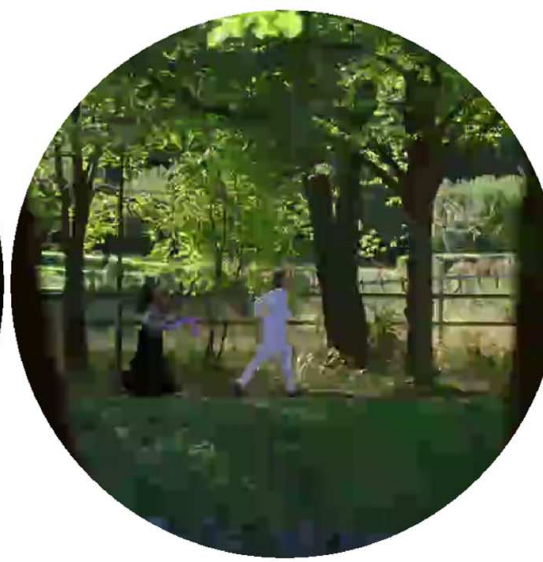

(k) Magnified macular image of the frame in Fig. 11(h). (c) Preprocessed by Lee et al. [29] at 0.024 bpp $(Q P=47)$, MOS $=2.24$.

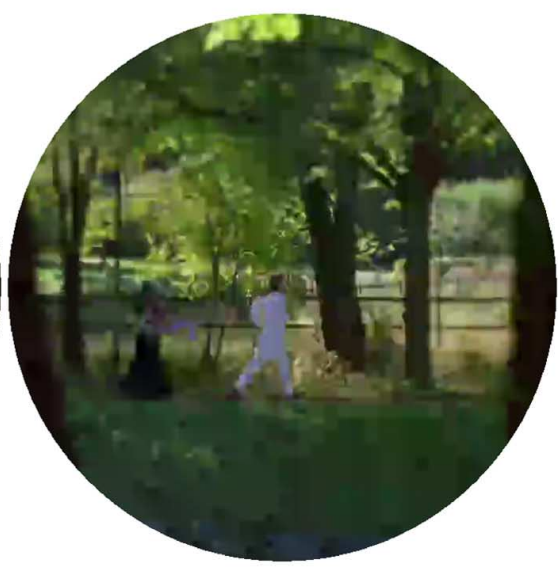

(f) Magnified macular image of the frame in Fig. 11(c).

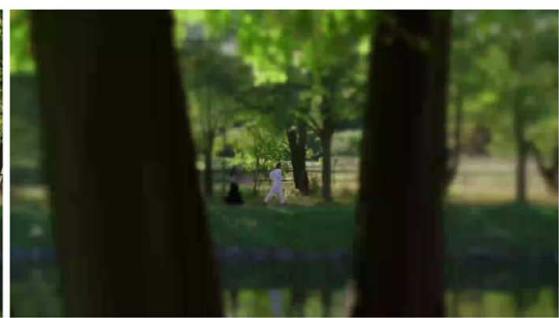

(i) Preprocessed by proposed method $(\Delta=15)$ at 0.022 bpp $(Q P=40), \operatorname{MOS}=2.70$.

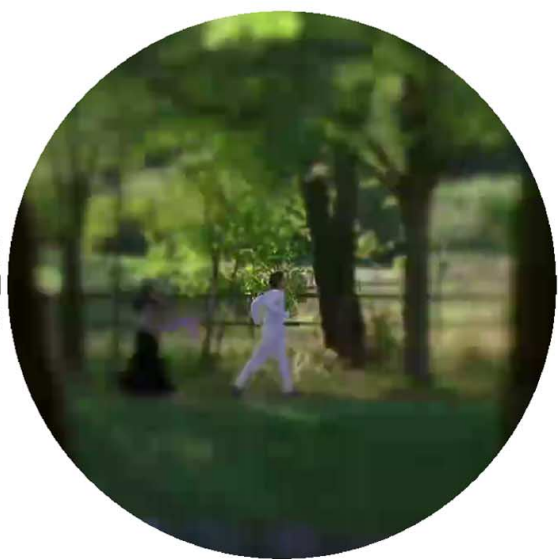

(1) Magnified macular image of the frame in Fig. 11(i).

Fig. 11. Compression results for the 10th frame of the 1080p sequence park joy, for $\Gamma=\Gamma_{1}=\Gamma_{2} \approx 92$ PPI, and $D \approx 22$ in. (a) Original. (b) At 0.029 bpp $(Q P=50), \mathrm{MOS}=1.61$. (c) Preprocessed by Lee et al. [29] at $0.024 \mathrm{bpp}(Q P=47)$, MOS $=2.24$. (d) Magnified macular image of the frame in Fig. 11(a). (e) Magnified macular image of the frame in Fig. 11(b). (f) Magnified macular image of the frame in Fig. 11(c). (g) Proposed method ( $\Delta=0)$. (h) At 0.049 bpp $(Q P=47), \operatorname{MOS}=2.42$. (i) Preprocessed by proposed method $(\Delta=15)$ at $0.022 \mathrm{bpp}(Q P=40)$, MOS $=2.70$. (j) Magnified macular image of the frame in Fig. 11(g). (k) Magnified macular image of the frame in Fig. 11(h). (1) Magnified macular image of the frame in Fig. 11(i).

we have a mean opinion score (MOS) and its $95 \%$ confidence interval for each compressed image.
As we can see from Table $\mathrm{V}$, the $\operatorname{MOS}$ of $C_{Q P_{i, j}}\left(T_{\Delta_{i, j}}\left(s_{i}\right)\right)$ is better than the MOS of $C_{50}\left(s_{i}\right)$ for every $i$, including 5 cases 
with statistical significance (for frames $s_{1}, s_{4}, s_{7}, s_{9}$, and $s_{14}$ ), even with a small subject pool of 10 people. We believe such results give enough psychological evidence to answer positively the last question of our problem statement, i.e., such preprocessing can make $Q(\tilde{y})>Q(\tilde{x})$ without increasing the bit rate. Moreover, the MOS of $C_{Q P_{i, j}}\left(T_{\Delta_{i, j}}\left(s_{i}\right)\right)$ is better than the MOS of $C_{Q P_{i}^{*}}\left(L\left(s_{i}\right)\right)$ in every case but one, including 2 cases with statistical significance (for frames $s_{7}$ and $s_{9}$ ), meaning that results regarding the proposed method can also be considered better than the ones provided by Lee et al. [29].

Finally, Fig. 11 shows results for the 10th frame of the 1080p sequence park joy. Again, we also displayed magnified macular images of such frames in order to facilitate the visualization of the results. The point $S$ for the 10th frame is located at the center of its macular image ${ }^{13}$.

As we can see from Fig. 11, the perceptual quality of the frames $s_{7}$ (Fig. 11(g)) and $T_{0}\left(s_{7}\right)$ (Fig. 11(d)) are arguably the same, especially when we look at their respective magnified macular images in Fig. 11(d) and 11(j). Moreover, although we can see a substantial amount of visual perceptual loss in Fig. 11(b), 11(c), 11(h), and 11(i) when compared to Fig. 11(a), when we look at the magnified macular images in Fig. 11(e), 11(f), 11(k), and 11(l) we can clearly see that the lattermost looks better than any of the first three, implying that the perceptual quality of the frame $C_{40}\left(T_{15}\left(s_{7}\right)\right.$ ) (Fig. 11(i)) can be considered better than the perceptual quality of the frames $C_{50}\left(s_{7}\right)$ (Fig. 11(b)), $C_{47}\left(L\left(s_{7}\right)\right)$ (Fig. 11(c)), and $C_{47}\left(s_{7}\right)$ (Fig. 11(h)). This means that the proposed method: can enhance the perceptual quality of an image under the bit rate restriction; can be considered better than the one provided by Lee et al. [29]; and, most importantly, while increasing the perceptual quality of a frame, can permit gains of approximately 2 times the compression ratio of a typical compression scheme.

\section{CONCLUSION}

The main objectives of finding a perceptually efficient way of representing images and determining its theoretical limitations were satisfactorily achieved. Under reasonable considerations, the proposed material combined with related theories successfully answered the three questions of our problem statement. Although we could use mostly background information to promptly answer the first of the three questions, showing that we can preprocess an image without changing its perceptual quality, the other two demanded more efforts from our end.

In order to answer the second question, we developed a theory that ultimately led to the proposed retina-based perceptually lossless limit. Such theory showed that the data in any image can be reduced to $E$ times its original amount with no visual perceptual loss. Results considering real devices showed that we can achieve data reduction ratios up to approximately $3: 1$, 9:1, and 16:1 with no visual perceptual loss under appropriate near, typical, and far viewing distances, respectively. In general, results showed that values for the perceptual efficiency of a display of real devices can be considered low.

In order to answer the last question, we developed a Gaussian foveation scheme with resolution control to process images prior to their transmissions. Such preprocessing method can

\footnotetext{
${ }^{13} S$ is at coordinates $(948,553)$.
}

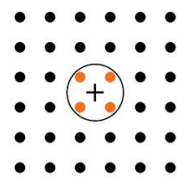

(a) $e_{D}=0.25$.

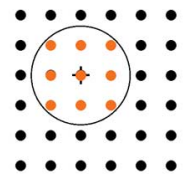

(b) $e_{D}=0 . \overline{1}$.

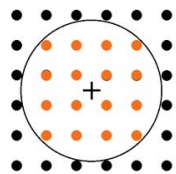

(c) $e_{D}=0.0625$
Fig. 12. Three different cases of image projection onto the retina, where a dot represent a pixel and a circle represent a cone. (a) $e_{D}=0.25$. (b) $e_{D}=0 . \overline{1}$. (c) $e_{D}=0.0625$.

be adjusted through a lossy parameter to fit limits on transmission bit rates. Results considering real images showed that the proposed preprocessing method can make $Q(\tilde{y})>Q(\tilde{x})$ without increasing the bit rates. Moreover, while increasing the perceptual quality, the proposed method achieved gains up to approximately 2 times the compression ratio of a typical compression scheme. However, both the enhancement of the perceptual quality and the gain relative to the compression ratio turned out to be highly dependent on the source complexity.

Finally, we believe as technology advances image sizes are likely to grow and display pixel densities tend to increase. Both factors contribute substantially to the benefits one can extract from the presented work, making it more valuable over the years. Overall, we believe the retina-based perceptually lossless limit and the Gaussian foveation scheme with resolution control proved to be powerful tools for compression purposes.

\section{FUTURE WORK}

Although we tried to be as complete as possible in our approach, a few questions remain open. Clearly, how to obtain a good estimation for the perceptual quality $Q$ of an image is one of them. In order to help answering such question, we want to make use of some previous works [47], [48]. Another important question we want to answer is whether or not it is possible to find an optimal value for the lossy parameter $\Delta$, and, in case it is, how to find it.

Additionally, we want to use information from subsequent stages of the human visual processing in future compression schemes. Ultimately, we have a strong desire to expand our research to late vision so that we can provide even more substantial contributions to the field.

\section{APPENDIX A \\ ON FiLTER STANDARD DEVIATION}

For the filter design, we need to describe its only parameter $\sigma_{p_{1} p_{2}}$ at every $P$. In order to do so, we can assume that $\sigma_{p_{1} p_{2}}$ is somehow related to $e_{D}\left[p_{1}, p_{2}\right]$. Then, we can analyze some specific cases to understand how we can use the information about $e_{D}\left[p_{1}, p_{2}\right]$ to determine $\sigma_{p_{1} p_{2}}$. In order to make our point more clear, we consider the cases shown in Fig. 12.

When $e_{D}\left[p_{1}, p_{2}\right]=(1 / M)$, it means that we have a proportion of $M$ pixels to 1 cone $^{14}$. In other words, it means that the signal is locally downsampled by a factor of $M$. So, considering the cases shown in Fig. 12, in order to avoid aliasing, we could locally filter the image by a separable ideal low-pass filter. Hence, the cutoff frequency of such filter would be $\pi \sqrt{e_{D}}$

\footnotetext{
${ }^{14}$ For points where $e_{D}\left[p_{1}, p_{2}\right]=q_{D}\left[p_{1}, p_{2}\right]$.
} 
in both horizontal and vertical directions. However, we are designing a Gaussian filter, and therefore we decided to approximate the 1-D filters by Gaussian filters whose Fourier transforms are of the form:

$$
H(\omega)=e^{-\frac{\omega^{2}}{2 \sigma_{\omega}^{2}}},
$$

where $\sigma_{\omega}$ is the standard deviation of such filter in the frequency domain.

In order to perform the approximation, we can use the $3 \mathrm{~dB}$ criteria to determine the cutoff frequency of the proposed filter, i.e., we can make $H\left(\omega_{c}\right)=(\sqrt{2} / 2)$, where $\omega_{c}$ is the cutoff frequency of the 1-D ideal low-pass filter. Hence:

$$
\sigma_{\omega}=\frac{\omega_{c}}{\sqrt{2 \ln \sqrt{2}}}
$$

Finally, considering only the cases shown in Fig. 12, and knowing that the relation between $\sigma_{n}$, the standard deviation of a Gaussian filter in the discrete-time domain, and $\sigma_{\omega}$, the standard deviation of such filter in the frequency domain, is given by $\sigma_{n}=\left(1 / \sigma_{\omega}\right)$, then:

$$
\sigma_{n}=\frac{1}{\pi} \sqrt{\frac{2 \ln \sqrt{2}}{e_{D}}} .
$$

For such cases, i.e., when $e_{D}=0.25, e_{D}=0 . \overline{1}$, and $e_{D}=$ 0.0625 , we have $\sigma_{n}=0.53, \sigma_{n}=0.8$, and $\sigma_{n}=1.06$, respectively. Also, we know that when $e_{D}=1, \sigma_{n}=0$ since no downsampling is performed and thus no filtering is required.

Now we can use those four values of $\sigma_{n}$ to estimate its value for all the other more challenging cases. In order to do so, we can choose a function that satisfactorily fits those four points and can be used to estimate $\sigma_{n}$ at all other points. We are interested in a function $f_{\sigma}\left(e_{D}\right)$ that equals 0 when $e_{D}=1$ and increases as $e_{D}$ decreases such that $f_{\sigma}\left(e_{D}\right) \leftarrow \infty$ as $e_{D} \leftarrow 0$. A function that fits well with our objective is $f_{\sigma}\left(e_{D}\right)=-k_{\sigma} \ln e_{D}$.

After fitting the data, we found that $k_{\sigma}=0.38$ minimized both the mean absolute error and the mean squared error between $f_{\sigma}\left(e_{D}\right)$ and $\sigma_{n}$ for those four points. Therefore, we finally made:

$$
\sigma_{p_{1} p_{2}}=-0.38 \ln e_{D}\left[p_{1}, p_{2}\right]
$$

\section{ACKNOWLEDGMENT}

The authors would like to thank Professor Daniel Simons for his crucial contributions regarding the subjective experiments and CAPES/Fulbright for sponsoring the research.

\section{REFERENCES}

[1] E. Hering, Outlines of a Theory of the Light Sense. Cambridge, MA, USA: Harvard Univ. Press, 1964.

[2] S. Shevell, The Science of Color. Amsterdam, The Netherlands: Elsevier, 2003.

[3] R. Blake and R. Sekuler, Perception. New York, NY, USA: McGrawHill, 2006.

[4] M. Farah, The Cognitive Neuroscience of Vision, ser. Fundamentals of cognitive neuroscience. Hoboken, NJ, USA: Blackwell, 2000.

[5] E. Rosen, "The invention of eyeglasses," J. History Med. Allied Sci., vol. 11 , no. 1 , pp. $13-46,1956$.
[6] E. Rosen, "The invention of eyeglasses," J. History Med. Allied Sci., vol. 11, no. 2, pp. 183-218, 1956.

[7] H. B. Leighton, "Frederic Eugene Ives, forgotten pioneer in color photography and the halftone," Image, vol. 19, no. 3, pp. 33-35, 1976.

[8] P. R. Jones, "Evolution of halftoning technology in the US patent literature," J. Electron. Imag., vol. 3, no. 3, pp. 257-275, 1994.

[9] A. Abramson, The History of Television, 1880 To 1941. Jefferson, NC, USA: McFarland, 2009.

[10] A. Abramson and C. Sterling, The History of Television, 1942 to 2000. Jefferson, NC, USA: McFarland, 2007.

[11] Z. Wang and A. C. Bovik, "Foveated image and video coding," in Digital Video, Image Quality and Perceptual Coding, H. R. Wu and K. R. Rao, Eds. Boca Raton, FL: CRC, 2006, ch. 14, pp. 431-457.

[12] L. Itti, "Automatic foveation for video compression using a neurobiological model of visual attention," IEEE Trans. Image Process., vol. 13, no. 10, pp. 1304-1318, Oct. 2004.

[13] Z. Wang and A. Bovik, "Embedded foveation image coding," IEEE Trans. Image Process., vol. 10, no. 10, pp. 1397-1410, Oct. 2001.

[14] Z. Wang, L. Lu, and A. Bovik, "Foveation scalable video coding with automatic fixation selection," IEEE Trans. Image Process., vol. 12, no. 2, pp. 243-254, Feb. 2003.

[15] S. Lee, M. Pattichis, and A. Bovik, "Foveated video compression with optimal rate control," IEEE Trans. Image Process., vol. 10, no. 7, pp. 977-992, Jul. 2001.

[16] K. Kaneko and N. Ohta, "4 K applications beyond digital cinema," in Proc. 16th Int. Conf. Virtual Syst. Multimedia (VSMM), 2010, pp. 133-136.

[17] T. Yamashita and K. Mitani, "8 K extremely-high-resolution camera systems," Proc. IEEE, vol. 101, no. 1, pp. 74-88, Jan. 2013.

[18] P. Burt, "Smart sensing within a pyramid vision machine," Proc. IEEE, vol. 76, no. 8, pp. 1006-1015, Aug. 1988.

[19] C. Bandera and P. Scott, "Foveal machine vision systems," in Proc. IEEE Int. Conf. Syst., Man, Cybern., 1989, vol. 2, pp. 596-599.

[20] A. Rojer and E. Schwartz, "Design considerations for a space-variant visual sensor with complex-logarithmic geometry," in Proc. 10th Int. Conf. Pattern Recogn., 1990, vol. ii, pp. 278-285, vol.2.

[21] Y. Y. Zeevi and R. Ginosar, "Foveating vision systems architecture. image acquisition and display," in Proc. SPIE-Int. Soc. Opt. Eng., 1990, vol. 1360 , pp. $371-377$, pt 1 .

[22] A. Califano, R. Kjeldsen, and R. Bolle, "Data and model driven foveation," in Proc. 10th Int. Conf. Pattern Recogn., 1990, vol. i, pp. $1-7$, vol.1

[23] M. Levoy and R. Whitaker, "Gaze-directed volume rendering," Comput. Graph. (ACM), vol. 24, pp. 217-223, 1990.

[24] C. F. R. Weiman, "Video compression via log polar mapping," in Proc. SPIE-Int. Soc. Opt. Eng., 1990, vol. 1295, pp. 266-277.

[25] P. L. Silsbee and A. C. Bovik, "Nonuniform visual pattern image sequence coding," in Proc. SPIE-Int. Soc. Opt. Eng., 1991, vol. 1452, pp. $409-419$.

[26] A. Basu and K. Wiebe, "Enhancing videoconferencing using spatially varying sensing," IEEE Trans. Syst., Man, Cybern. A: Syst. Humans, vol. 28, no. 2, pp. 137-148, Mar. 1998.

[27] S. Lee, A. C. Bovik, and B. L. Evans, "Efficient implementation of foveation filtering," in Proc. Texas Instrum. DSP Educator's Conf., Houston, TX, USA, Aug. 1999.

[28] S. Lee, M. Pattichis, and A. Bovik, "Foveated video quality assessment," IEEE Trans. Multimedia, vol. 4, no. 1, pp. 129-132, Mar. 2002.

[29] S. Lee and A. Bovik, "Fast algorithms for foveated video processing," IEEE Trans. Circuits Syst. Video Technol., vol. 13, no. 2, pp. 149-162, Feb. 2003

[30] S. Lee, C. Podilchuk, V. Krishnan, and A. C. Bovik, "Foveation-based error resilience and unequal error protection over mobile networks," $J$. VLSI Signal Process. Syst. Signal, Image, Video Technol., vol. 34, no. $1-2$, pp. 149-166, 2003.

[31] S. Lee, A. C. Bovik, and Y. Y. Kim, "High quality, low delay foveated visual communications over mobile channels," J. Vis. Commun. Image Represent., vol. 16, no. 2, pp. 180-211, 2005.

[32] C.-C. Ho, J.-L. Wu, and W.-H. Cheng, "A practical foveation-based rate-shaping mechanism for mpeg videos," IEEE Trans. Circuits Syst. Video Technol., vol. 15, no. 11, pp. 1365-1372, Nov. 2005.

[33] B. Guenter, M. Finch, S. Drucker, D. Tan, and J. Snyder, "Foveated 3D graphics," ACM Trans. Graphics, vol. 31, no. 6, pp. 1-22, 2012. 
[34] K. H. Lee and P. C. Chung, "An attention emphasized bit arrangement in 3-D SPIHT video coding for human vision," J. Visual Commun. Image Represent., vol. 24, no. 3, pp. 255-269, 2013.

[35] H. Oh and W. Kim, "Video processing for human perceptual visual quality-oriented video coding," IEEE Trans. Image Process., vol. 22, no. 4, pp. 1526-1535, Apr. 2013.

[36] D. Pamplona and A. Bernardino, "Smooth foveal vision with gaussian receptive fields," in Proc. 9th IEEE-RAS Int. Conf. Humanoid Robots Humanoids , 2009, pp. 223-229.

[37] R. S. Wallace, P. Ong, B. B. Bederson, and E. L. Schwartz, "Space variant image processing," Int. J. Comput. Vis., vol. 13, no. 1, pp. 71-90, 1994.

[38] C. A. Curcio, K. R. Sloan, R. E. Kalina, and A. E. Hendrickson, "Human photoreceptor topography," J. Compar. Neurol., vol. 292, no. 4, pp. 497-523, 1990.

[39] C. A. Curcio and K. A. Allen, "Topography of ganglion cells in human retina," J. Compar. Neurol., vol. 300, no. 1, pp. 5-25, 1990.

[40] F. D. De Monasterio and P. Gouras, "Functional properties of ganglion cells of the rhesus monkey retina," J. Physiol., vol. 251, no. 1, pp. 167-195, 1975.

[41] N. R. Council, Advances in the Modularity of Vision Selections From a Symposium on Frontiers of Visual Science. Washington, DC: National Academies Press, 1990.

[42] Y. Zeevi and E. Shlomot, "Nonuniform sampling and antialiasing in image representation," IEEE Trans. Signal Process., vol. 41, no. 3, pp. 1223-1236, Mar. 1993.

[43] M. A. Webster and R. L. De Valois, "Relationship between spatialfrequency and orientation tuning of striate-cortex cells.," J. Opt. Soc. Amer. A, vol. 2, no. 7, pp. 1124-1132, 1985.

[44] T. Fujine, Y. Kikuchi, M. Sugino, and Y. Yoshida, "Real-life in-home viewing conditions for flat panel displays and statistical characteristics of broadcast video signal," Jpn. J. Appl. Phys. Part 1: Regular Papers and Short Notes and Review Papers, vol. 46, no. 3 B, pp. 1358-1362, 2007.

[45] G. E. Legge and C. A. Bigelow, "Does print size matter for reading? a review of findings from vision science and typography," $J$. Vis., vol. 11 , no. 5, 2011.

[46] A. Bovik, Handbook of Image and Video Processing, ser. Communications, Networking and Multimedia. Amsterdam, The Netherlands: Elsevier, 2010.

[47] A. Ciancio, A. L. N. Targino da Costa, E. A. B. da Silva, A. Said, R. Samadani, and P. Obrador, "Objective no-reference image blur metric based on local phase coherence," Electron. Lett., vol. 45, no. 23, pp. 1162-1163, 2009.

[48] A. Ciancio, A. L. N. Targino da Costa, E. A. B. da Silva, A. Said, R. Samadani, and P. Obrador, "No-reference blur assessment of digital pictures based on multifeature classifiers," IEEE Trans. Image Process., vol. 20, no. 1, pp. 64-75, Jan. 2011.

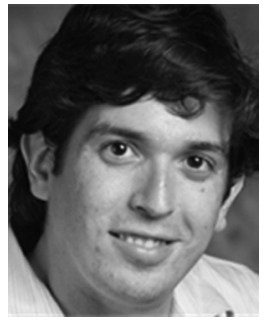

André Luiz N. Targino da Costa received his B.Sc. degree in Electronics and Computer Engineering (Magna Cum Laude) and his M.Sc. degree in Electrical Engineering from the Federal University of Rio de Janeiro (UFRJ), Brazil, in 2006 and 2009, respectively. He is currently pursuing his Ph.D. degree in Electrical and Computer Engineering at the University of Illinois at Urbana-Champaign. His research interests include Wearables, Gesture Recognition, Haptics, Perceptual Processing (especially Visual and Haptic), Signal Processing (especially Image and Video), Computer Vision, Information Theory, Compression (especially Image and Video), Artificial Intelligence, and Neural Networks. Mr. Targino da Costa received a Best Student Award in Electronics and Computer Engineering from the UFRJ in 2006 and a CAPES/Fulbright Fellowship in 2009. He is a member of the IEEE and was also part of the team that developed the Brazilian Digital Television System.

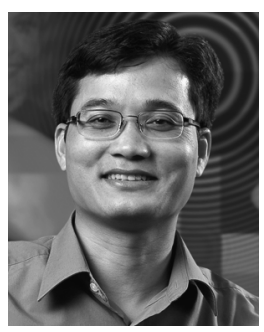

Minh N. Do (M'01-SM'07-F'14) was born in Vietnam in 1974. He received the B.Eng. degree in computer engineering from the University of Canberra, Australia, in 1997, and the Dr.Sci. degree in communication systems from the Swiss Federal Institute of Technology Lausanne (EPFL), Switzerland, in 2001.

Since 2002, he has been on the faculty at the University of Illinois at Urbana-Champaign (UIUC), where he is currently an Associate Professor in the Department of Electrical and Computer Engineering, and hold joint appointments with the Coordinated Science Laboratory, the Beckman Institute for Advanced Science and Technology, and the Department of Bioengineering. His research interests include image and multi-dimensional signal processing, wavelets and multiscale geometric analysis, computational imaging, augmented reality, and visual information representation.

He received a Silver Medal from the 32nd International Mathematical Olympiad in 1991, a University Medal from the University of Canberra in 1997, a Doctorate Award from the EPFL in 2001, a CAREER Award from the National Science Foundation in 2003, and a Young Author Best Paper Award from IEEE in 2008. He was named a Beckman Fellow at the Center for Advanced Study, UIUC, in 2006, and received of a Xerox Award for Faculty Research from the College of Engineering, UIUC, in 2007. He was a member of the IEEE Signal Processing Theory and Methods Technical Committee, Image, Video, and Multidimensional Signal Processing Technical Committee, and an Associate Editor of the IEEE TRANSACTIONS ON IMAGE PROCESSING. 